\title{
Impact of non-hydrostatic effects and trapped lee waves on mountain wave drag in directionally sheared flow
}

Article

Accepted Version

Yu, C. L. and Teixeira, M. A. C. (2015) Impact of nonhydrostatic effects and trapped lee waves on mountain wave drag in directionally sheared flow. Quarterly Journal of the Royal Meteorological Society, 141 (690). pp. 1572-1585. ISSN 1477-870X doi: https://doi.org/10.1002/qj.2459 Available at https://centaur.reading.ac.uk/37629/

It is advisable to refer to the publisher's version if you intend to cite from the work. See Guidance on citing.

Published version at: http://dx.doi.org/10.1002/aj.2459

To link to this article DOI: http://dx.doi.org/10.1002/qj.2459

Publisher: Royal Meteorological Society

All outputs in CentAUR are protected by Intellectual Property Rights law, including copyright law. Copyright and IPR is retained by the creators or other copyright holders. Terms and conditions for use of this material are defined in the End User Agreement.

www.reading.ac.uk/centaur 
Central Archive at the University of Reading

Reading's research outputs online 


\title{
Impact of non-hydrostatic effects and trapped lee waves on mountain wave drag in directionally sheared flow
}

\author{
C. L. Yu and M. A. C. Teixeira* \\ Department of Meteorology, University of Reading, Reading RG6 6BB, UK
}

${ }^{*}$ Correspondence to: Miguel A. C. Teixeira, Department of Meteorology, University of Reading, Earley Gate, PO Box 243, Reading RG6 6BB, UK. Email: M.A.Teixeira@reading.ac.uk

The orographic gravity wave drag produced in flow over an axisymmetric mountain when both vertical wind shear and non-hydrostatic effects are important was calculated using a semi-analytical two-layer linear model, including unidirectional or directional constant wind shear in a layer near the surface, above which the wind is constant. The drag behaviour is determined by partial wave reflection at the shear discontinuity, wave absorption at critical levels (both of which exist in hydrostatic flow), and total wave reflection at levels where the waves become evanescent (an intrinsically nonhydrostatic effect), which produces resonant trapped lee wave modes. As a result of constructive or destructive wave interference, the drag oscillates with the thickness of the constant-shear layer and the Richardson number within it $(R i)$, generally decreasing at low $R i$ and when the flow is strongly non-hydrostatic. Critical level absorption, which increases with the angle spanned by the wind velocity in the constant-shear layer, shields the surface from reflected waves, keeping the drag closer to its hydrostatic limit. While, for the parameter range considered here, the drag seldom exceeds this limit, a substantial drag fraction may be produced by trapped lee waves, particularly when the flow is strongly non-hydrostatic, the lower layer is thick and $R i$ is relatively high. In directionally sheared flows with $R i=O(1)$, the drag may be misaligned with the surface wind in a direction opposite to the shear, a behaviour which is totally due to non-trapped waves. The trapped lee wave drag, whose reaction force on the atmosphere is felt at low levels, may therefore have a distinctly different direction from the drag associated with vertically propagating waves, which acts on the atmosphere at higher levels.

Key Words: $\quad$ flow over orography; gravity wave drag; non-hydrostatic effects; trapped lee waves; directional wind shear; critical levels; wave reflection; resonance

This article has been accepted for publication and undergone full peer review but has not been through the copyediting, typesetting, pagination and proofreading process, which may lead to differences between this version and the Version of Record. Please cite this article as doi: $10.1002 / \mathrm{qj} .2459$

This article is protected by copyright. All rights reserved. 


\section{Introduction}

The parametrization of mountain waves in weather and climate prediction models run at global scales remains a key scientific issue at present (Stensrud,2009), and is expected to remain so for the foreseeable future. Among the numerous processes that occur at scales below the grid resolution in these models, but have a substantial impact on the resolved flow, mountain wave drag is thought to be especially relevant at horizontal scales of $\mathrm{O}(10$ $\mathrm{km})$, for typical values of the atmospheric parameters (Gill,1982). This force is also important at larger scales, where mountain waves become influenced by the rotation of the Earth, and at smaller scales, where they are affected by non-hydrostatic effects (Teixeira et al.,2008b).

Mountain waves influenced by rotation are already adequately represented at the resolutions currently employed operationally in meteorological models, but that is not the case with nonhydrostatic mountain waves, which have typical horizontal wavelengths of a few km (Wurtele et al.,1987; Keller,1994). Linear theory suggests that the drag produced by such waves becomes progressively less relevant as their horizontal scale decreases, both because they are forced by relatively narrow, and therefore relatively low mountains or hills, and because a larger fraction of the waves becomes evanescent, being unable to transport momentum. However, this latter assertion is based on results from linear wave theory for flow with constant wind and static stability (Gill,1982; Teixeira et al.,2008b), and it is not obvious whether it holds for vertically sheared flows. Indeed, previous studies have suggested that non-trivial interactions may take place between different physical processes (for example, wave nonlinearity and non-hydrostatic effects - see Durran (1986), or nonlinearity and rotation - see Ólafsson and Bougeault (1997)), leading to higher values of the drag than expected. That possibility will be investigated in the present study for the conjugated effects of vertical wind shear and non-hydrostaticity.

It has been shown recently that the drag produced by nonhydrostatic mountain waves in atmospheres where the wind and static stability vary vertically can receive a substantial contribution from resonant trapped modes, which do not exist for constant atmospheric parameters (Teixeira et al. 2013a,2013b). Whereas vertically propagating waves have a continuous spectrum, and decelerate the atmospheric flow at high elevations, trapped lee waves have a discrete spectrum, for which the drag may be calculated separately (Smith,1976), and they decelerate the atmosphere at low levels. The piecewise-constant atmospheric parameter profiles assumed by Teixeira et al. (2013a) and Teixeira et al. (2013b) are representative to a certain degree of fast variations in static stability or wind speed at a given height, allowing an easy evaluation of trapped lee wave drag. But they are realistic to a limited extent, since one of the most common reasons for wave trapping is a continuous increase of the wind speed with height (Grubišić and Stiperski,2009; Stiperski and Grubišić,2011). In that situation, the dynamics of the trapped lee waves is considerably more complicated, among other reasons because the wave-trapping height is not unique, depending instead on the wavenumber.

Despite the importance of vertical wind shear in, for example, momentum deposition in the high atmosphere (which directly leads to a deceleration of the large-scale flow) (Shutts and Gadian,1999), expressions for the surface drag in gravity wave parametrizations adopted in the most modern weather and climate prediction models still neglect (for simplicity) both wind shear and non-hydrostatic effects (Lott and Miller,1997; Kim and Doyle,2005). However, the wave trapping mechanism mentioned above conjugates these two effects. It is clear, then, that more knowledge is necessary about non-hydrostatic mountain waves with wind shear, particularly concerning the behaviour of the associated drag force.

Corrections to the drag due to vertical wind shear for wind profiles with a relatively slow variation have been derived by Teixeira and Miranda (2006) using a WKB approximation, in an analytical form easy to implement in drag parametrizations, but only in hydrostatic conditions. The hydrostatic assumption allows these corrections to be independent of the detailed orography shape, as long as this is assumed to be axisymmetric (Teixeira et al.,2004), 2D (Teixeira and Miranda,2004), or have an elliptical horizontal cross-section (Teixeira and Miranda,2006).

There have been relatively few theoretical studies addressing the drag produced by non-hydrostatic mountain waves, and fewer still considering the additional effect of directional shear, due 
to the complexity of the corresponding wave solutions. Wurtele et al. (1987) and Keller (1994), for example, considered a single-layer atmosphere where the wind increases linearly, or a two-layer atmosphere where the wind increases linearly in the lower layer and becomes constant in the upper layer, but they limited their treatment to unidirectional shear flows and did not focus on the drag. Shutts (1995), on the other hand, considered a constant-shear flow with directional shear extending indefinitely with height, but he only evaluated its impact on the drag in the hydrostatic approximation. However, while flows with directional shear are ubiquitous in the real atmosphere, shear layers that extend indefinitely lead to unrealistically high winds that artificially induce total wave trapping. Nevertheless, the relevance of non-hydrostatic effects for drag parametrization will continue to increase as the resolution of meteorological models improves without being able to resolve the entire spectrum of internal gravity waves.

For all these reasons, in the present study the joint effects of vertical wind shear and non-hydrostaticity will be addressed using an inviscid linear semi-analytical two-layer model where the wind has constant unidirectional or directional shear in a lower layer and constant velocity in the upper layer. This model, which extends the calculations of Teixeira et al. (2008a) to nonhydrostatic conditions, will be used to evaluate mountain wave drag, focusing in particular on its partition between contributions coming from vertically propagating waves and from trapped lee waves.

Since, as far as we know, this is the first time the behaviour of the drag produced by non-hydrostatic mountain waves in directional shear flow is systematically investigated, nonlinear effects will be neglected for simplicity. Durran (1986) showed that nonlinearity enhances the amplitude of trapped lee waves by a large factor when these are much shorter than the width of the mountains that generate them, but his results were for 2D flow. In the flows over an axisymmetric mountain to be addressed in the present study, the effect of nonlinearity is likely to be considerably weaker (cf. Miranda and James,1992; Miranda and Valente,1997), due to directional wave dispersion (Teixeira et al.,2008a).

Boundary layer effects are also neglected here, to achieve a cleaner model setup that is manageable mathematically and easier This article is protected by copyright. All rights reserved. to interpret physically. In reality, some overlap between the impact of trapped lee wave drag and of turbulent boundary layer drag is expected, since both forces act at low levels. In very general terms, boundary layers are known to lower the amplitude of mountain waves and thus the drag associated with them (Ólafsson and Bougeault,1996; Jiang et al.,2008), but more complex interactions may take place (Lott,2007).

The remainder of this paper is organized as follows. Section 2 provides a description of the semi-analytical model adopted to tackle this problem, as well as its verification against numerical simulations of trapped lee waves. The contribution of trapped lee waves to the drag is also analyzed for flow over a $2 \mathrm{D}$ ridge. In Section 3 the main results are presented, focusing on the drag behaviour in flow over an axisymmetric mountain (the canonical type of orography usually chosen for illustrating the 3D effects occurring in flows with directional shear). Finally, in Section 4 the main findings of this study are summarized.

\section{Semi-analytical model}

We consider a two-layer linear model of atmospheric flow over a 3D axisymmetric mountain. In the lower layer, the background wind has constant unidirectional or directional shear, whereas the wind magnitude and direction become constant in the upper layer. The mountain width may be varied, allowing to control the intensity of non-hydrostatic effects. Wave reflections, which may generate resonant wave modes, may either be partial at the shear discontinuity existing at the interface between the two layers, or total at the levels where the waves change from vertically propagating to evanescent (an intrinsically non-hydrostatic effect). This model extends those of Wurtele et al. (1987) and Keller (1994) (which did not consider directional wind shear), that of Shutts (1995) (which considered a constant-shear layer extending indefinitely), and that of Teixeira et al. (2008a) (which assumed hydrostatic flow). Clearly, considering an infinite shear layer, as done by Shutts (1995), although allowing interesting insights, is unrealistic in an unbounded atmosphere, even in hydrostatic conditions (as shown by Teixeira et al. (2008a)). But it becomes especially so when non-hydrostatic effects are taken into account, because then all vertically propagating waves launched by the orography, if they are not absorbed by critical levels, become 
evanescent (and therefore trapped) at some height (Wurtele et al.,1987;Keller,1994).

\subsection{Analytical solutions}

Stationary flow over an isolated mountain is considered, so both the terrain elevation $h(x, y)$ and all flow perturbations associated with the mountain waves (including the vertical velocity perturbation $w$ and pressure perturbation $p$ ) can be expressed as Fourier integrals (Lin,2007)

$$
\begin{aligned}
& w(\mathbf{x})=\int_{-\infty}^{\infty} \int_{-\infty}^{\infty} \hat{w}\left(k_{1}, k_{2}, z\right) e^{i \mathbf{k} \cdot \mathbf{x}} d k_{1} d k_{2}, \\
& p(\mathbf{x})=\int_{-\infty}^{\infty} \int_{-\infty}^{\infty} \hat{p}\left(k_{1}, k_{2}, z\right) e^{i \mathbf{k} \cdot \mathbf{x}} d k_{1} d k_{2}, \\
& h(x, y)=\int_{-\infty}^{\infty} \int_{-\infty}^{\infty} \hat{h}\left(k_{1}, k_{2}\right) e^{i \mathbf{k} \cdot \mathbf{x}} d k_{1} d k_{2},
\end{aligned}
$$

where the hat denotes Fourier transform, $\mathbf{x}=(x, y, z)$ and $\mathbf{k}=$ $\left(k_{1}, k_{2}\right)$ is the horizontal wavenumber vector.

Under the assumptions of inviscid, non-rotating flow and lowamplitude waves, the equations of motion with the Boussinesq approximation may be combined, and the wave motion is described by the Taylor-Goldstein equation (Nappo,2012), which is written in terms of the Fourier transform of the vertical velocity $\hat{w}$

$$
\hat{w}^{\prime \prime}+\left\{\frac{N^{2} k_{12}^{2}}{(\mathbf{U} \cdot \mathbf{k})^{2}}-\frac{\mathbf{U}^{\prime \prime} \cdot \mathbf{k}}{\mathbf{U} \cdot \mathbf{k}}-k_{12}^{2}\right\} \hat{w}=0
$$

where $\mathbf{U}(z)=(U, V)$ is the background wind vector, the magnitude of the horizontal wavenumber is $k_{12}=\left(k_{1}^{2}+k_{2}^{2}\right)^{1 / 2}$ and the primes denote differentiation with respect to height, $z$. Note that, to a first approximation, non-Boussinesq effects may still be taken into account using (4), provided that $w$ is viewed as a vertical velocity scaled by density, in which case the 'real' vertical velocity is given by $\left(\rho_{0} / \rho\right)^{1 / 2} w$ (where $\rho(z)$ is the reference density at a given level and $\rho_{0}$ its value at the surface) (see Shutts and Gadian,1999). As we are considering stationary flow, the phase velocity $\mathbf{c}$ is automatically set to zero and dropped from (4). Please refer to equation (A1) in Appendix A for a non-stationary version

It will be assumed that both layers in the model have the same Brunt-Väisälä frequency, $N$. The constant shear of the wind profile in the lower layer is described by the shear vector $\boldsymbol{\alpha}=$ This article is protected by copyright. All rights reserved. where $U_{0}$ is the surface wind and $H$ is the height of the lower layer. In (5), the background wind at the surface is aligned with $x$ (without any loss of generality due to the axisymmetry of the orography to be considered). A set of scenarios with different shear directions are presented in Section 3. Because the wind always either varies linearly or is constant, the curvature terms in (4) vanish. So, in the lower layer, $z \in(0, H)$, that equation becomes

$$
\hat{w}^{\prime \prime}+\left[\frac{N^{2} k_{12}^{2}}{\left\{U_{0} k_{1}+(\boldsymbol{\alpha} \cdot \mathbf{k}) z\right\}^{2}}-k_{12}^{2}\right] \hat{w}=0
$$

The height of critical levels, which by definition are levels where $\mathbf{U}\left(z_{c}\right) \cdot \mathbf{k}=0$ and where (4) becomes singular, can be written for the wind profile (5) as $z_{c}=-U_{0} k_{1} /(\boldsymbol{\alpha} \cdot \mathbf{k})$. Note that, in general (i.e. unless $\boldsymbol{\alpha}$ is aligned in the $x$-direction), $z_{c}$ depends on the orientation angle of $\mathbf{k}$. Critical levels only have an impact on the waves, and hence on the drag, when they are located within the atmosphere, $z_{c}>0$, i.e. when $U_{0} k_{1}$ and $\boldsymbol{\alpha} \cdot \mathbf{k}$ have opposite signs. On the other hand, turning points, where the coefficient between square brackets in (6) changes sign, and the waves shift from vertically propagating to evanescent or vice-versa, occur for the wind profile (5) at the height $z=\left( \pm N-U_{0} k_{1}\right) /(\boldsymbol{\alpha} \cdot \mathbf{k})$. Clearly, this height is always a function of the wavenumber, even if the flow is unidirectional $\left(\alpha_{2}=0\right)$. While it defines the level at which waves of a given wavenumber will be trapped, it does not guarantee that those waves will be resonant, which will only happen if an additional condition is fulfilled. This dependence of the trapping height on the wavenumber is one of the aspects in which the present model (as those of Wurtele et al. (1987), Keller (1994) and Shutts (1995)) differs from the simpler models of Scorer (1949) and Teixeira et al. (2013a), where the trapping height is fixed at a single level by a discontinuity in the Scorer parameter.

The Richardson number of the background flow in the lower layer is by definition $R i=N^{2} /|\boldsymbol{\alpha}|^{2}$, but a modified Richardson 
number may be written $\tilde{R} i=N^{2} k_{12}^{2} /(\boldsymbol{\alpha} \cdot \mathbf{k})^{2}$, which is obtained from $R i$ by replacing $\boldsymbol{\alpha}$ with its projection along $\mathbf{k}$. Clearly, $\tilde{R} i \geq R i$, and this definition has the advantage that (6) may be expressed in a similar form as for $2 \mathrm{D}$ mountain waves:

$$
\hat{w}^{\prime \prime}+\left\{\frac{\tilde{R} i}{\left(z-z_{c}\right)^{2}}-k_{12}^{2}\right\} \hat{w}=0 .
$$

The above linear second-order differential equation can be transformed into a modified Bessel equation of pure complex order (Booker and Bretherton,1967). For $z>z_{c}$, solutions to (7) corresponding to upward and downward propagating wave energy can be written in terms of the modified Bessel function $I$,

$$
\begin{aligned}
\hat{w}^{\uparrow}(\xi) & =\sqrt{\xi} I_{i(\operatorname{sgn}) \mu}\left(k_{12} \xi\right), \\
\hat{w}^{\downarrow}(\xi) & =\sqrt{\xi} I_{-i(\operatorname{sgn}) \mu}\left(k_{12} \xi\right),
\end{aligned}
$$

where $\xi=z-z_{c}>0, \mu=\sqrt{\tilde{R} i-0.25}, \uparrow \downarrow$ denotes the direction of wave energy propagation and $\operatorname{sgn}=\operatorname{sign}\left(\mathbf{U}^{\prime}\left(z_{c}\right) \cdot \mathbf{k}\right)=$ $\operatorname{sign}(\boldsymbol{\alpha} \cdot \mathbf{k})$. The extension of this pair of solutions across the critical level is simple by using the properties of the Bessel $I$ function and introducing a small imaginary phase speed. The general result, for $z<z_{c}(\xi<0)$, is

$$
\begin{aligned}
& \hat{w}^{\uparrow}(\xi)=-i(\operatorname{sgn}) e^{\pi \mu} \sqrt{|\xi|} I_{i(\operatorname{sgn}) \mu}\left(k_{12}|\xi|\right), \\
& \hat{w}^{\downarrow}(\xi)=-i(\operatorname{sgn}) e^{-\pi \mu} \sqrt{|\xi|} I_{-i(\operatorname{sgn}) \mu}\left(k_{12}|\xi|\right) .
\end{aligned}
$$

A detailed justification for the above solutions is presented in Appendix A. Shutts (1995) performed a similar extension of his wave solution across the critical level but the factor $i$, which corresponds to a phase shift, was missing in his final expression (between his Eqs. (32) and (33)). Equations (10)-(11) correspond to waves whose energy propagates in the vertical direction sufficiently near to their critical levels (where the flow is perfectly hydrostatic, and hence no evanescent waves exist), but become evanescent at the turning points where the coefficient between brackets in (7) becomes negative, as explained before.

A drawback of employing this pair of solutions is that they both exhibit exponential growth when they enter the evanescent region, which is not practical for numerical calculations. This problem can be avoided by expressing the solutions instead in terms of the modified Bessel function $K_{i \mu}$ and the related function $L_{i \mu}$ introduced by Wurtele et al. (1987), both of imaginary order, where $K_{i \mu}$ is pure exponentially decaying and $L_{i \mu}$ is exponentially growing beyond the turning points delimiting evanescent regions. This pair of solutions has been employed by Wurtele et al. (1987) and Keller (1994) in treatments that included non-Boussinesq effects, which was important for filtering out long waves and their associated resonant modes in the single layer-model of Wurtele et al. (1987). However, in the two-layer model adopted here, the upper layer already prevents those longwave resonances by allowing the propagation of long waves, so non-Boussinesq effects (beyond those that can be accommodated by using the density scaling mentioned above) are excluded for simplicity. $K_{i \mu}$ and $L_{i \mu}$ are both real functions for real arguments, and physically correspond to the interference between upward and downward propagating waves. The relation between these two sets of solutions is relegated to Appendix A, where it is shown that an alternative form for (8)-(9) and (10)-(11) using $K_{i \mu}$ and $L_{i \mu}$ is:

$$
\begin{gathered}
\hat{w}^{\uparrow}(\xi)= \begin{cases}\sqrt{\xi}\left\{L_{i \mu}\left(k_{12} \xi\right)-i(\operatorname{sgn}) K_{i \mu}\left(k_{12} \xi\right)\right\} & z>z_{c} \\
-i(\operatorname{sgn}) e^{\pi \mu} \sqrt{|\xi|}\left\{L_{i \mu}\left(k_{12}|\xi|\right)-i(\operatorname{sgn}) K_{i \mu}\left(k_{12}|\xi|\right)\right\} & z<z_{c}\end{cases} \\
\hat{w}^{\downarrow}(\xi)= \begin{cases}\sqrt{\xi}\left\{L_{i \mu}\left(k_{12} \xi\right)+i(\operatorname{sgn}) K_{i \mu}\left(k_{12} \xi\right)\right\} & z>z_{c} \\
-i(\operatorname{sgn}) e^{-\pi \mu} \sqrt{|\xi|}\left\{L_{i \mu}\left(k_{12}|\xi|\right)+i(\operatorname{sgn}) K_{i \mu}\left(k_{12}|\xi|\right)\right\} & z<z_{c} .\end{cases}
\end{gathered}
$$

This article is protected by copyright. All rights reserved. 


$$
w(z=0)=\mathbf{U}_{1}(z=0) \cdot \nabla_{H} h(\mathbf{x}),
$$

In the two-layer model introduced above, the correct wave solution in the lower layer is a linear combination of the solutions described previously, satisfying certain boundary conditions. For the upper layer, the wind profile is constant, which allows the following exact solution

$$
\hat{w}(z>H)=C \exp (i m z)
$$

where $C$ is some complex coefficient, and the vertical wavenumber $m$ is defined as follows

$$
m=\left\{\begin{array}{ll}
\frac{k_{12} \sqrt{N^{2}-\left(\mathbf{U}_{H} \cdot \mathbf{k}\right)^{2}}}{\mathbf{U}_{H} \cdot \mathbf{k}} & \text { if } \frac{N^{2} k_{12}^{2}}{\left(\mathbf{U}_{H} \cdot \mathbf{k}\right)^{2}}-k_{12}^{2}>0 \\
i \sqrt{k_{12}^{2}-\frac{N^{2} k_{12}^{2}}{\left(\mathbf{U}_{H} \cdot \mathbf{k}\right)^{2}}} & \text { if } \frac{N^{2} k_{12}^{2}}{\left(\mathbf{U}_{H} \cdot \mathbf{k}\right)^{2}}-k_{12}^{2}<0
\end{array},\right.
$$

where $\mathbf{U}_{H}=\left(U_{H}, V_{H}\right)=\mathbf{U}(z=H)$ is the wind vector in the upper layer. This definition of $m$ satisfies the far-field radiation boundary condition (Teixeira et al.,2004) or the boundedness condition as $z \rightarrow+\infty$, depending on whether the wave is propagating or evanescent. As a result, the complete solution in the entire atmosphere is

$$
\hat{w}= \begin{cases}A \hat{w}^{\uparrow}+B \hat{w}^{\downarrow} & \text { for } 0<z<H \\ C \exp (i m z) & \text { for } z>H,\end{cases}
$$

where $A$ and $B$ are also complex coefficients.

Three constraints are required to solve for these three unknowns. By denoting the wind velocity in the lower layer as $\mathbf{U}_{1}(z)$, the free-slip or zero-normal-flow boundary condition can where $\nabla_{H}=(\partial / \partial x, \partial / \partial y)$ is the horizontal gradient operator. Taking the Fourier transform of (17) and using also (16), the boundary condition for $\hat{w}$ at the surface is

$$
A \hat{w}^{\uparrow}(0)+B \hat{w}^{\downarrow}(0)=i\left(\mathbf{U}_{1}(0) \cdot \mathbf{k}\right) \hat{h}(\mathbf{k}) .
$$

The continuity of the vertical velocity at the interface between the two layers can be expressed as

$$
A \hat{w}^{\uparrow}(H)+B \hat{w}^{\downarrow}(H)-C e^{i m H}=0 .
$$

Finally, the continuity of pressure at the same interface implies

$$
\begin{aligned}
& A\left\{\left(\mathbf{U}_{1}^{\prime}(H) \cdot \mathbf{k}\right) \hat{w}^{\uparrow}(H)-\left(\mathbf{U}_{1}(H) \cdot \mathbf{k}\right)\left(\hat{w}^{\uparrow}\right)^{\prime}(H)\right\} \\
+ & B\left\{\left(\mathbf{U}_{1}^{\prime}(H) \cdot \mathbf{k}\right) \hat{w}^{\downarrow}(H)-\left(\mathbf{U}_{1}(H) \cdot \mathbf{k}\right)\left(\hat{w}^{\downarrow}\right)^{\prime}(H)\right\} \\
+ & C\left(\mathbf{U}_{H} \cdot \mathbf{k}\right) i m e^{i m H}=0 .
\end{aligned}
$$

This last condition makes use of the relation between the pressure and the vertical velocity,

$$
\hat{p}=i \frac{\rho_{0}}{k_{12}^{2}}\left\{\left(\mathbf{U}^{\prime} \cdot \mathbf{k}\right) \hat{w}-(\mathbf{U} \cdot \mathbf{k}) \hat{w}^{\prime}\right\} .
$$

which can be obtained by taking the horizontal divergence of the momentum equations and applying the Fourier transform (Teixeira et al.,2004). The three unknown coefficients $A, B$ and $C$ can then be obtained using Cramer's rule, which yields the following expressions:

$$
\begin{array}{lc}
A=i \hat{h}\left(\mathbf{U}_{1}(0) \cdot \mathbf{k}\right) \frac{1}{\hat{w}_{0}^{\uparrow}-\gamma \hat{w}_{0}^{\downarrow}}, & B=-i \hat{h}\left(\mathbf{U}_{1}(0) \cdot \mathbf{k}\right) \frac{\gamma}{\hat{w}_{0}^{\uparrow}-\gamma \hat{w}_{0}^{\downarrow}}, \\
C=i \hat{h}\left(\mathbf{U}_{1}(0) \cdot \mathbf{k}\right) \frac{\Gamma_{H}\left\{\left(\hat{w}_{H}^{\uparrow}\right)^{\prime} \hat{w}_{H}^{\downarrow}-\left(\hat{w}_{H}^{\downarrow}\right)^{\prime} \hat{w}_{H}^{\uparrow}\right\} e^{-i m H}}{\hat{w}_{0}^{\uparrow}\left\{\hat{w}_{H}^{\downarrow}\left(\Gamma_{H} i m+\Gamma_{H}^{\prime}\right)-\left(\hat{w}_{H}^{\downarrow}\right)^{\prime} \Gamma_{H}\right\}-\hat{w}_{0}^{\downarrow}\left\{\hat{w}_{H}^{\uparrow}\left(\Gamma_{H} i m+\Gamma_{H}^{\prime}\right)-\left(\hat{w}_{H}^{\uparrow}\right)^{\prime} \Gamma_{H}\right\}},
\end{array}
$$


where

$$
\gamma=\frac{\hat{w}_{H}^{\uparrow}\left(\Gamma_{H} i m+\Gamma_{H}^{\prime}\right)-\left(\hat{w}_{H}^{\uparrow}\right)^{\prime} \Gamma_{H}}{\hat{w}_{H}^{\downarrow}\left(\Gamma_{H} i m+\Gamma_{H}^{\prime}\right)-\left(\hat{w}_{H}^{\downarrow}\right)^{\prime} \Gamma_{H}}, \quad \Gamma(z)=\mathbf{U}_{1}(z) \cdot \mathbf{k},
$$

and the subscripts 0 or $H$ of $\hat{w}^{\uparrow \downarrow}$ and $\Gamma$ mean that those functions are evaluated at the heights $z=0$ or $z=H$, respectively.

\subsection{Drag calculation}

The formal definition of surface drag gives this force as the integral of the pressure gradient force over the orography (Teixeira et al.,2004),

$$
\mathbf{D}=\int_{-\infty}^{\infty} \int_{-\infty}^{\infty} p(z=0) \nabla_{H} h(x, y) d x d y
$$

Using (2) and (3), (24) can be expressed alternatively as

$$
\mathbf{D}=4 \pi^{2} i \int_{-\infty}^{\infty} \int_{-\infty}^{\infty} \mathbf{k} \overline{\left.\hat{p}^{(} z=0\right)} \hat{h}\left(k_{1}, k_{2}\right) d k_{1} d k_{2}
$$

where the overbar denotes complex-conjugate (Teixeira et al.,2004). This definition is convenient, since the wave solutions obtained in Fourier space can be used directly in the drag calculation, which reduces the computational cost and improves the accuracy of the result. In Section 3, all surface drag yalues will be normalized by the drag $D_{0}$, valid for hydrostatic flow with infinite $R i$ (i.e. a constant wind profile),

$$
D_{0}=4 \pi^{3} \rho_{0} N\left|\mathbf{U}_{0}\right| \int_{0}^{\infty} k_{12}^{2}|\hat{h}|^{2} d k_{12}
$$

where $\mathbf{U}_{0}=\mathbf{U}_{1}(0)$.

While the solutions to the mountain wave problem in Fourier space are analytical, being expressed in terms of Bessel functions (as was shown above), the fields of flow perturbations in physical space and the drag must be calculated numerically. The challenging aspect of these calculations is that (unlike in hydrostatic conditions) the wave spectrum contains both a continuous part and a discrete part (corresponding to resonant trapped modes). More details about these calculations are provided below. (a)

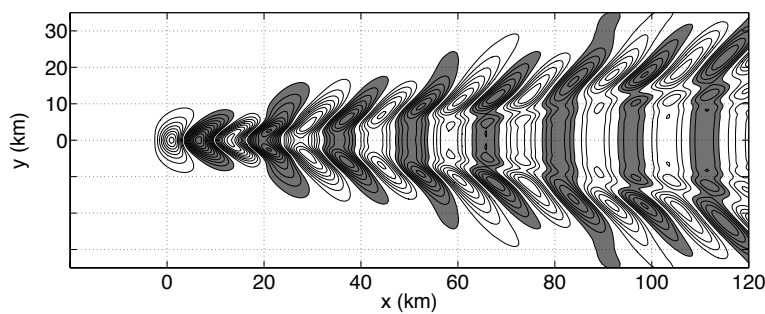

(b)

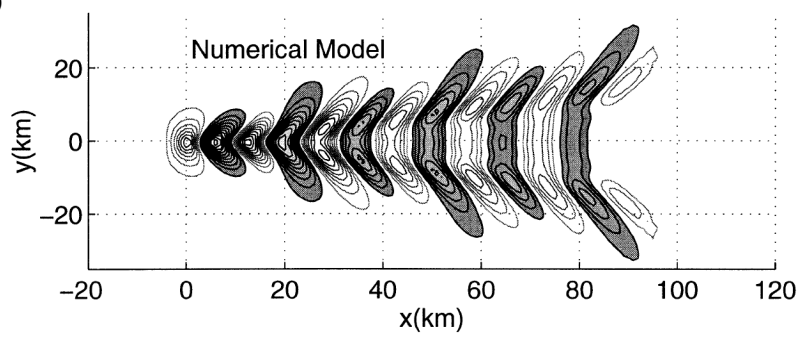

Figure 1. Density-scaled vertical velocity at a height $z=2.5 \mathrm{~km}$ (a) from the present model, and (b) from the numerical simulations of Broutman et al. (2003) (reproduced from their Figure 6). The contour interval is $0.02 \mathrm{~m} \mathrm{~s}^{-1}$, positive values are shaded and the zero contour is omitted for clear visualization. Note that sponge layers were applied beyond $x=80 \mathrm{~km}$ and above $z=35 \mathrm{~km}$ in the simulations of Broutman et al. (2003).

\subsection{Accuracy of the model: a three-dimensional example}

An example is given next to illustrate how well the present model captures the resonant wave modes. The mean wind assumed in this example has a unidirectional forward shear aligned in the $x$-direction. There is no critical level within the atmosphere, so a pronounced trapped lee wave pattern is expected to occur. The wind profile and surface elevation in the two-layer model are defined as,

$$
\begin{array}{cc}
(U, V)=\left(U_{0}+\alpha_{1} z, 0\right) & \text { for } z<H, \\
(U, V)=\left(U_{H}, 0\right)=\left(U_{0}+\alpha_{1} H, 0\right) & \text { for } z \geq H, \\
h(x, y)=\frac{h_{0}}{\left\{1+(x / a)^{2}+(y / a)^{2}\right\}^{3 / 2}},
\end{array}
$$

where $h_{0}$ and $a$ are, respectively, the height and half-width of the axisymmetric bell-shaped mountain defined by (29). Broutman et al. (2003) carried out numerical simulations using a similar orography and wind profile, but with an unbounded shear layer, and a sponge layer above $z=35 \mathrm{~km}$. In order to compare the present model with their results, the following parameters are adopted:

$$
\begin{gathered}
U_{0}=10 \mathrm{~m} \mathrm{~s}^{-1}, \quad \alpha_{1}=0.0025 \mathrm{~s}^{-1}, \quad H=28 \mathrm{~km}, \\
a=2.5 \mathrm{~km}, \quad h_{0}=100 \mathrm{~m}, \quad N=0.01 \mathrm{~s}^{-1} .
\end{gathered}
$$

This article is protected by copyright. All rights reserved. 
It can be shown from (30)-(31) that $U_{H}=80 \mathrm{~m} \mathrm{~s}^{-1}$ and $R i=$ $N^{2} / \alpha_{1}^{2}=16$. Although $U_{H}$ is perhaps unrealistically high, it should be kept in mind that we aim here to study the theoretical properties of the flow, rather than reproduce a real atmospheric situation, so the thickness of the lower layer is designed to be large enough for the two models to be comparable.

Figure 1(a) shows the density-scaled vertical velocity field $w(x, y)$ at a height $z=2.5 \mathrm{~km}$ from the present model, and Figure

1(b) shows similar results from the numerical simulations of Broutman et al. (2003) (reproduced from their Figure 6, bottom). The trapped lee wave pattern is very clear, and the two results are in very good agreement, with only small differences at the core of the maxima and minima near the obstacle. These differences are likely due to the different number of layers employed in the two models: in the two-layer model wave reflection (even if weak) may occur at $z=H$, while in the single-layer numerical simulations of Broutman et al. (2003) any upward propagating waves should be absorbed by the sponge layer existing above $z=35 \mathrm{~km}$. Additionally, numerical integration errors in either model could also be a contributing factor. Further downstream of the obstacle, the discrepancies between the two models first become larger, and then suddenly overwhelming, which is clearly due to the sponge layer used beyond $x=80 \mathrm{~km}$ in the numerical simulations of Broutman et al. (2003).

In Figure 1, the trapped lee wave train downstream of the mountain results from singularities at the resonant wavenumbers in the wave solution. This poses challenges in the numerical integration used to obtain the pressure field or the drag. In all calculations in this paper, a weak dissipation is introduced through addition of a small imaginary wavenumber $i \mathbf{k} \delta$ to the solutions, with $\delta=0.001$. This leads to a very slight decay of the wave perturbations downstream of the mountain, limiting the extent of the trapped lee wave train. Through this procedure, singularities are smoothed, and numerical integration in the wavenumber domain can be performed more easily. Note that, since the dissipation magnitude is proportional to the magnitude of the wavenumber, for trapped lee waves with wavelength $O(10$ $\mathrm{km}$ ) (as observed in Figure 1), the magnitude of the dominant imaginary wavenumber is $2 \pi \delta / 10^{4} \approx 6 \times 10^{-7} \mathrm{~m}^{-1}$, which gives an e-folding distance for the flow decay of $1.5 \times 10^{3} \mathrm{~km}$. This This article is protected by copyright. All rights reserved. is high enough to render the flow pattern within the domain of interest indistinguishable from its inviscid counterpart. To make the integration over all wavenumbers more accurate, an adaptive grid spacing for $\mathbf{k}$ is adopted, which is refined near the resonant wavenumbers, to ensure that errors in numerical integration do not exceed a specified bound.

\subsection{The drag contribution from resonant wave modes}

As seen in the preceding section, an important feature of nonhydrostatic effects is the ability to create resonant wave modes, which correspond to long trains of trapped lee waves. Since this study focuses on the calculation of the surface drag, the contribution of trapped lee waves to this force is of great importance. This aspect can be understood most easily by examining the surface pressure field created by $2 \mathrm{D}$ trapped lee waves in a single-layer atmosphere.

By using 1D versions of (2) and (21), and adopting an alternative but equivalent form for the first of these equations to facilitate the discussion, the surface pressure perturbation may be calculated as

$$
p_{0}(x)=2 \rho_{0} \operatorname{Re}\left[\int_{0}^{\infty} \frac{i}{k_{1}}\left(U_{0}^{\prime} \hat{w}_{0}-U_{0} \hat{w}_{0}^{\prime}\right) e^{i k_{1} x} d k_{1}\right]
$$

where the subscripts 0 of $p_{0}$ and $\hat{w}_{0}$ denote their evaluation at $z=0$. The above equation uses the one-sided Fourier transform, which is valid for real quantities such as $p(x, z)$. Note that, in this form, only positive wavenumbers are involved in the integration. Suppose that $\hat{w}\left(k_{1}, z\right)$ can be expressed as $F \hat{\phi}\left(k_{1}, z\right)$ for some constant $F$ and function $\hat{\phi}$. In order to satisfy the lower boundary condition $\hat{w}_{0}\left(k_{1}\right)=i \hat{h}\left(k_{1}\right) U_{0} k_{1}, \quad F=i \hat{h} U_{0} k_{1} / \hat{\phi}\left(k_{1}, z=0\right)$ is required, except at wavenumbers $k_{1}=k_{j}$ for which $\hat{\phi}_{0}\left(k_{j}\right) \equiv$ $\hat{\phi}\left(k_{j}, z=0\right)=0$. This corresponds to resonant wave modes where singularities exist. At the resonant wavenumber $k_{j}, F$ could be non-zero even for zero topographic forcing, $\hat{h}=0$. Following 
this reasoning, (32) can be rewritten as

$$
\begin{aligned}
& p_{0}(x)=2 \rho_{0} \operatorname{Re}\left[\int_{0}^{\infty}\left[-U_{0}^{\prime} U_{0} \hat{h}+U_{0}^{2} \frac{\hat{\phi}_{0}^{\prime}\left(k_{1}\right)}{\hat{\phi}_{0}\left(k_{1}\right)} \hat{h}\right] e^{i k_{1} x} d k_{1}\right] \\
& =I_{1}+I_{2} \\
& \text { where } I_{1}=-2 \rho_{0} U_{0}^{\prime} U_{0} \operatorname{Re}\left[\int_{0}^{\infty} \hat{h} e^{i k_{1} x} d k_{1}\right] \text {, } \\
& I_{2}=2 \rho_{0} U_{0}^{2} \operatorname{Re}\left[\int_{0}^{\infty} \hat{h} \frac{\hat{\phi}_{0}^{\prime}\left(k_{1}\right)}{\hat{\phi}_{0}\left(k_{1}\right)} e^{i k_{1} x} d k_{1}\right] \text {. }
\end{aligned}
$$

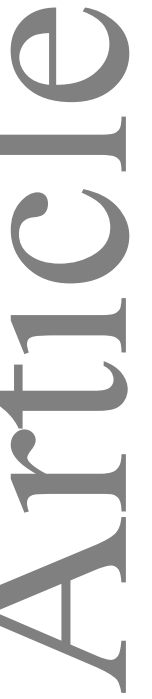

The first integral $I_{1}$ is proportional to the mountain profile $h(x)$, and hence gives no contribution to the drag. Following the pioneering study of Scorer (1949) (see also Sawyer (1960) and Mitchell et al. (1990)), the second integral $I_{2}$ can be split into two terms, which may be evaluated using contour integration,

$$
I_{2}=\left\{\begin{array}{rr}
2 \rho_{0} U_{0}^{2} \operatorname{Re}\left(\int_{C_{1}} \hat{h} \frac{\hat{\phi}_{0}^{\prime}\left(k_{1}\right)}{\hat{\phi}_{0}\left(k_{1}\right)} e^{i k_{1} x} d k_{1}\right) & \text { for } x>0 \\
+2 \pi i \sum_{j} L_{j} & \\
2 \rho_{0} U_{0}^{2} \operatorname{Re}\left(\int_{C_{2}} \hat{h} \frac{\hat{\phi}_{0}^{\prime}\left(k_{1}\right)}{\hat{\phi}_{0}\left(k_{1}\right)} e^{i k_{1} x} d k_{1}\right) & \text { for } x<0 .
\end{array}\right.
$$

The two integration paths $C_{1}$ and $C_{2}$, as illustrated in Figure 2, are designed to make the integrals decay to zero far away from the mountain, and $L_{j}$ is the residue at the pole corresponding to the resonance wavenumber $k_{j}$,

$$
L_{j}=2 i \rho_{0} U_{0}^{2} \hat{h}\left(k_{j}\right) \sin \left(k_{j} x\right) \frac{\hat{\phi}^{\prime}\left(k_{j}, z=0\right)}{\frac{\partial \hat{\phi}}{\partial k_{1}}\left(k_{j}, z=0\right)} .
$$

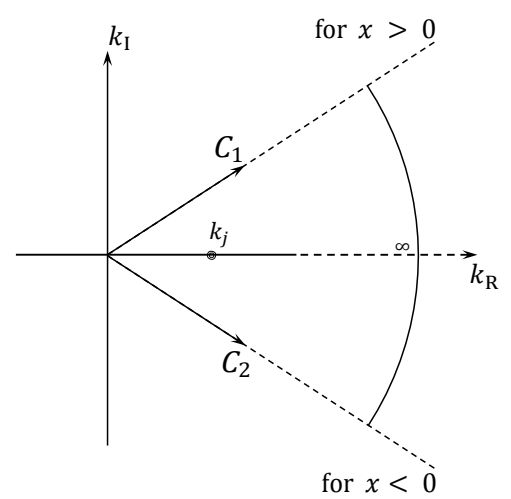

Figure 2. Integration paths $C_{1}$ and $C_{2}$ used in the contour integrals of (34), in complex wavenumber space, where $k_{1}=k_{R}+i k_{I}$. Adapted from Scorer (1949).
The surface pressure perturbation can then be divided into two parts: the near-field pressure and the far-field pressure. The nearfield pressure receives contributions from the integral $I_{1}$, plus the complex contour integral $I_{2}$, while the far-field pressure only receives contributions from resonant wave modes.

If we assume that the surface elevation corresponds to a 2D bell-shaped mountain, i.e. $h(x)=h_{0} /\left\{1+(x / a)^{2}\right\}$, then its Fourier transform is simply $\hat{h}\left(k_{1}\right)=\left(h_{0} a / 2\right) \exp \left(-a\left|k_{1}\right|\right)$. If the mean wind has a linear forward shear that extends indefinitely (so that $z_{c}<0$ ), then the wave solution satisfying the boundedness condition must be $\hat{\phi}=\sqrt{z-z_{c}} K_{i \mu}\left(k_{1}\left(z-z_{c}\right)\right)$, in agreement with the treatment of Wurtele et al. (1987). By substituting this expression into (35), the contribution of the residues to the farfield surface pressure is found to be

$$
p_{0}(x \gg a)=-\frac{2 \pi \rho_{0} U_{0}^{2} h_{0} a}{\left|z_{c}\right|} \sum_{j} e^{-a k_{j}} k_{j} \sin \left(k_{j} x\right),
$$

which can also be derived using Eq. (7) of Wurtele et al. (1987). Although the above expression is for the far-field pressure (or the pressure due to resonant trapped lee wave modes), its contribution is in fact valid for all $x>0$, as shown by (34). Note also from (36) that for each resonant wave mode $j$, the coefficient multiplying the sine function is always negative (since only positive values of $k_{j}$ are considered). Therefore, the resonant pressure components always deepen further the low pressure existing on the lee side of the orography. Hence, from the definition of drag (24), the contribution of resonant trapped lee wave modes to the surface drag must be positive. This is consistent with the findings of Teixeira et al. (2013a,2013b) for atmospheres with a simpler structure.

Another relevant result is that under the assumption of a wind profile with forward shear extending indefinitely, the near-field pressure does not give a contribution to the surface drag, i.e. in addition to $I_{1}$, the complex contour integral $I_{2}$ excluding the singularity is symmetric with respect to the mountain. This is demonstrated in Appendix B. Consequently, it can be concluded that if there is no leakage of gravity waves to the upper atmosphere (i.e. all the waves are reflected back to the surface), the pressure due to non-resonant wave modes gives no contribution to the drag. This result (which is consistent with the findings of Bretherton

This article is protected by copyright. All rights reserved. 
(a)

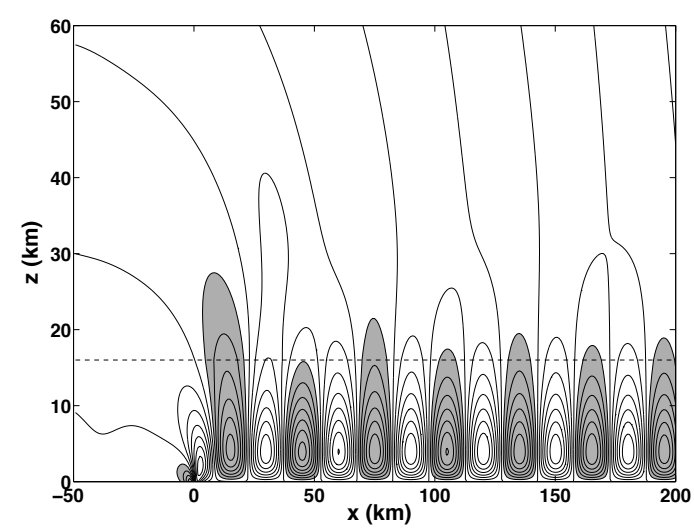

(b)

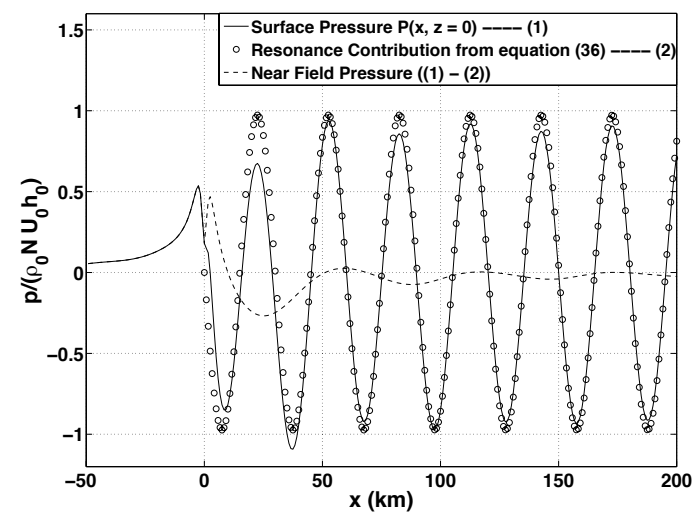

Figure 3. Flow over a 2D mountain ridge of width $a=2.5 \mathrm{~km}$ and height $h_{0}=$ $500 \mathrm{~m}$ using a two-layer model that approximates a single-layer atmosphere. $N=$ $0.01 \mathrm{~s}^{-1}$ for both layers, $U_{0}=10 \mathrm{~m} \mathrm{~s}^{-1}$, and the height of the lower layer is $H=16 \mathrm{~km}$ with $U_{H}=90 \mathrm{~m} \mathrm{~s}^{-1}$, implying $R i=4$. (a) Density-scaled vertical velocity $w(x, z)$ (contour spacing $0.25 \mathrm{~m} \mathrm{~s}^{-1}$ ); (b) surface pressure perturbation (normalized by $\rho_{0} N U_{0} h_{0}$ ) and its components (see legend).

(1969)) might not seem surprising, given that the drag is only produced by waves that are able to propagate energy away from the mountain and, for such a wind profile, all those waves correspond to resonant trapped modes. But one must keep in mind that the orography would also be able, in principle, to launch waves with a continuous range of wavenumbers that can only propagate near the surface. However, those waves cancel out through destructive interference.

Figure 3 shows an example of flow over a 2D mountain ridge produced by the two-layer model adopted here, in a situation that tries to approximate a single-layer atmosphere. The wind has a forward shear $\alpha_{1}=5 \times 10^{-3} \mathrm{~s}^{-1}$, the surface wind speed is $U_{0}=$ $10 \mathrm{~ms}^{-1}$ and the lower layer thickness is $H=16 \mathrm{~km}$. Under these conditions, the wind speed at the top of the lower layer is high $\left(U_{H}=90 \mathrm{~ms}^{-1}\right)$, preventing leakage to the upper layer of all but the lowest wavenumbers $\left(\mathrm{Na} / U_{H}=0.28\right)$ and causing only weak wave reflection at $z=H(R i=4$ in the lower layer).

This is confirmed in Figure 3(a) by the fact that the tops of the trapped lee wave cells are located near the top of the lower layer (denoted by the horizontal dashed line), and the wave activity in the upper layer (both associated with the propagation of long waves and extension of trapped lee waves into the upper layer) is weak. Therefore, the lower layer appears to be thick enough to contain most of the significant wave energy, and the two-layer model can approximate a single-layer model. Additionally, for these parameter values, only one resonant wave mode is produced, as is clearly shown by the regular shape of the cells of upward and downward motion in the $w$ field on the lee side of the mountain (Figure 3(a)). The associated surface pressure is plotted in Figure 3(b), together with its near-field and resonant components. Note that the density-scaled vertical velocity $w$ presented in Figure 3(a) can differ by a large factor from the 'true' vertical velocity at high levels, for example, at $z=20 \mathrm{~km}$, this factor is $\left(\rho_{0} / \rho\right)^{1 / 2} \approx 3.7$ according to the US Standard Atmosphere (1976).

As downstream distance from the mountain increases in Figure $3(\mathrm{~b})$, the surface pressure disturbance swiftly converges to the resonant surface pressure predicted by (36). Moreover, by subtracting the resonant pressure contribution from the total surface pressure perturbation, we obtain an approximate form for the near-field pressure, which is seen to be almost symmetric with respect to the mountain (see the dashed line near $x \gtrsim 0$ and solid line near $x \lesssim 0$ in Figure 3(b)). The contribution of the near-field pressure to the surface drag is in this case quite small, and most of the drag is due to the resonant mode. The small departure is due to the fact that the shear does not really extend indefinitely, so some weak wave reflection at $z=H$ and some leakage of gravity waves to the upper layer are allowed to take place (a lowamplitude gravity wave with a long wavelength is visible in Figure 3(a) propagating at $z>H$ ).

\section{Results and Discussion}

In the linear approximation, the two-layer atmosphere introduced previously can be fully described by four non-dimensional parameters, namely: the direction of shear, which may be quantified by $\alpha_{2} / \alpha_{1}$; the ratio of the wind magnitude in the upper layer to that at the surface $\left|\mathbf{U}_{H}\right| /\left|\mathbf{U}_{0}\right|$; the Richardson number $R i$ in the lower layer; and the non-dimensional width of the mountain 
Table 1. Parameters used in the two-layer model

\begin{tabular}{|c|c|c|c|c|c|c|}
\hline Case & $N\left(\mathrm{~s}^{-1}\right)$ & $\mathbf{U}_{0}\left(\mathrm{~ms}^{-1}\right)$ & Figure numbers & $\hat{a}=N a /\left|\mathbf{U}_{0}\right|$ & $\tan ^{-1}\left(\frac{\alpha_{2}}{\alpha_{1}}\right)$ & $\frac{\mid \mathbf{U}_{H}}{\left|\mathbf{U}_{0}\right|}$ \\
\hline Lin4(I), Lin8(I) & 0.01 & $(10,0)$ & 5,6 & Hydro, $8,4,2,1.5$ & 0 & 4,8 \\
\hline $\operatorname{Lin}(\mathrm{II})$ & 0.01 & $(10,0)$ & $8(a), 9(a, b), 10(a, b)$ & Hydro, $8,4,2,1.5$ & $\pi / 4$ & 4 \\
\hline $\operatorname{Lin}(\mathrm{III})$ & 0.01 & $(10,0)$ & $8(\mathrm{~b}), 9(\mathrm{c}, \mathrm{d}), 10(\mathrm{c}, \mathrm{d})$ & Hydro, $8,4,2,1.5$ & $\pi / 2$ & 4 \\
\hline Lin(IV) & 0.01 & $(10,0)$ & $8(\mathrm{c}), 9(\mathrm{e}, \mathrm{f})$ & Hydro, $8,4,2,1.5$ & $3 \pi / 4$ & 4 \\
\hline $\operatorname{Lin}(\mathrm{V})$ & 0.01 & $(10,0)$ & 7 & Hydro, $8,4,2,1.5$ & $\pi$ & 4 \\
\hline
\end{tabular}

(a)

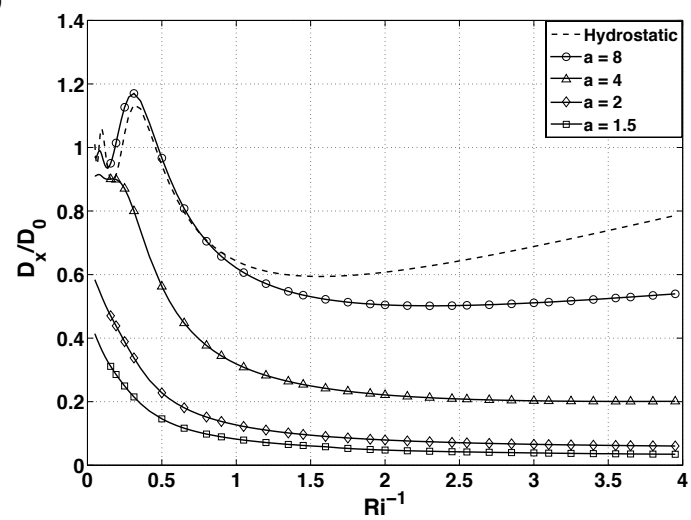

(b)

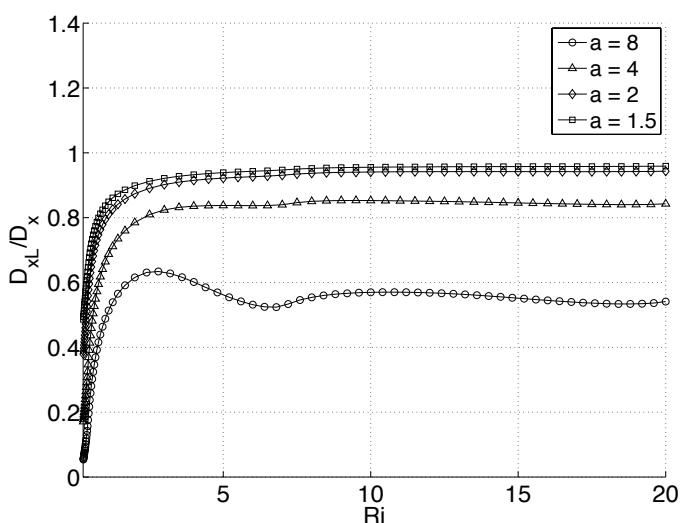

.
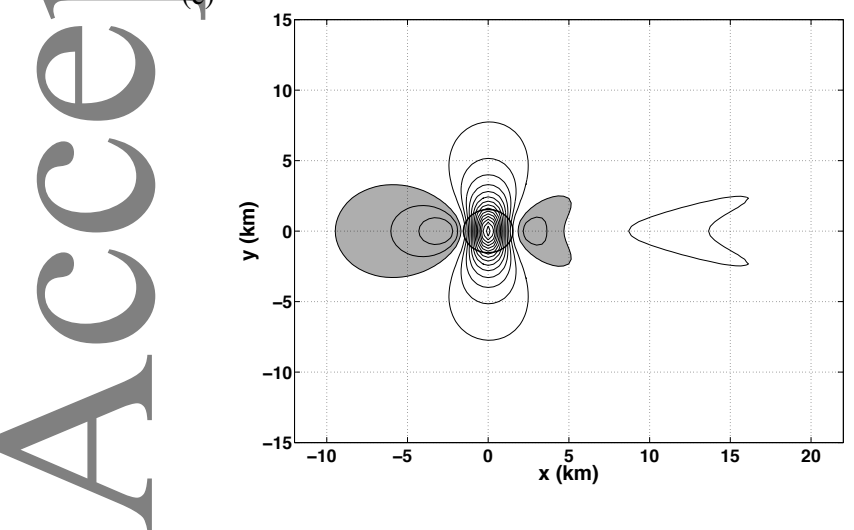

Figure 6. (a),(b): similar to Figure 5, but for the wind profile Lin8(I); (c) normalized surface pressure $p(z=0) /\left(\rho_{0} N U_{0} h_{0}\right)$ for the same flow with $\hat{a}=2$ and $R i=$ 0.5 . The contour spacing is 0.06 , with the zero contour omitted for clarity. Shaded contours: positive values. The circle centred at $(0,0)$ denotes $0.5 h_{0}$ surface elevation.

but become so as the wind speed increases in the lower layer (an essentially non-hydrostatic effect), may also cause a reduction in the drag magnitude through destructive wave interference. These

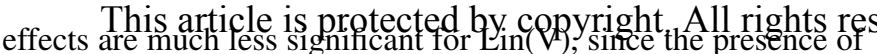

the total critical level in this wind profile filters a large portion of the waves, reducing the impact of reflected waves at the surface (Teixeira et al.,2008a). This happens both because the wind speed decreases with height below the critical level, suppressing resonant trapped modes in that region, and because above the critical level trapped modes, as well as waves reflected at the shear discontinuity, are partially absorbed by the critical level as they propagate downwards. As a result, the drag reduction as $\hat{a}$ decreases is more significant for $\operatorname{Lin} 4(\mathrm{I})$ or $\operatorname{Lin} 8(\mathrm{I})$ than for $\operatorname{Lin}(\mathrm{V})$, even when $R i$ is very large. This is shown by the fact that the drag magnitudes for $\hat{a}=2$ and 1.5 , for example, in cases Lin4(I) (Figure 5(a)) and Lin(V) (Figure 7) (which both assume $\left.\left|U_{H} / U_{0}\right|=4\right)$ do not approach the same limits as $R i \rightarrow \infty$ (these are approximately 0.6 and 0.4 in the first case, but 0.73 and 0.6 in the second).

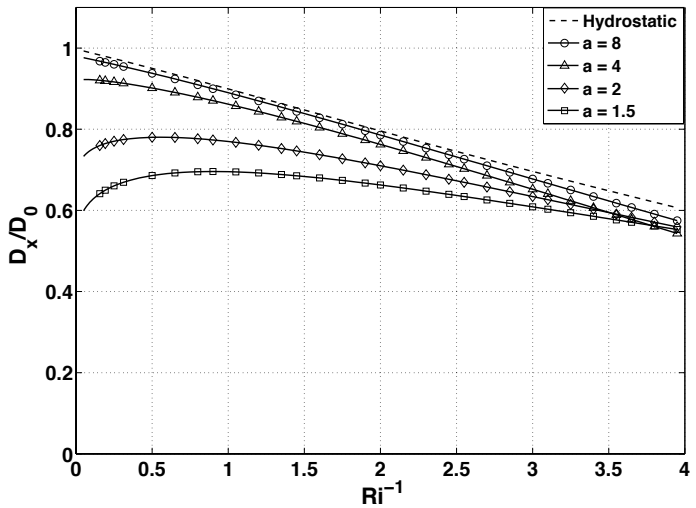

Figure 7. Similar to Figure 5(a), but for the wind profile Lin(V). The trapped lee wave drag contribution is not shown, as it is insignificant in this case.

Another important difference can be seen in the drag variation for small values of $\hat{a}$ when $R i$ is low: in cases $\operatorname{Lin} 4(\mathrm{I})$ and Lin8(I), the drag magnitude decreases to a small value and then becomes constant as $R i^{-1}$ increases, while in case $\operatorname{Lin}(\mathrm{V})$ it increases slightly and then decreases gradually. The decrease in drag magnitude in Lin4(I) and Lin8(I) probably happens because the number of resonant wave modes in the lower layer is reduced when $R i$ decreases, as mentioned by Keller (1994). This is consistent with the behaviour of the drag specifically associated 
with trapped lee waves, displayed in Figures 5-6(b) (the trapped lee wave drag in case $\operatorname{Lin}(\mathrm{V})$ is lower than 0.06 , so it was omitted). Indeed, when $R i$ decreases below 2 (in Figure 5(b)) or 1 (in Figure 6(b)), the resonant modes are weak, and so is their contribution to the drag. As a result, for these values of $R i$ the drag is mainly associated with the near-field pressure, which gives only a small contribution to it, for reasons explained in Section 2.5. This is especially so when the lower atmospheric layer is thick (i.e. $\left|U_{H} / U_{0}\right|$ is large). Then, most of the waves become evanescent and are totally reflected before they reach $z=H$ (because $N a /\left|\mathbf{U}_{H}\right|$, which is a measure of non-hydrostatic effects at that level, is low), so the near field pressure is quite symmetric with respect to the mountain (see Figure 6(c)). As shown in Figure 6(a), the drag magnitude for the case where $\left|U_{H} / U_{0}\right|=8$ decreases to an even smaller value than when $\left|U_{H} / U_{0}\right|=4$ if $\hat{a}=2$ or 1.5 and $R i$ is low. The trapped lee wave train shown in the surface pressure field of Figure 6(c) is consistent with this picture, being very weak and decaying over a short distance to the lee side of the mountain. And the pressure perturbation is indeed dominated by an approximately symmetric near-field component.

\subsection{Directional shear - Lin(II), Lin(III) and Lin(IV)}

In the interest of brevity, results will only be presented for the wind profiles $\operatorname{Lin}(\mathrm{II}), \operatorname{Lin}(\mathrm{III})$ and $\operatorname{Lin}(\mathrm{IV})$ for $\left|\mathbf{U}_{H}\right| /\left|\mathbf{U}_{0}\right|=4$. The way in which the results are affected for other values of this parameter, for example $\left|\mathbf{U}_{H}\right| /\left|\mathbf{U}_{0}\right|=8$, is essentially the same as illustrated in the preceding section for Lin8(I). The directional shear in these wind profiles produces a critical layer in the atmosphere, within which every level is the critical level for a certain wavenumber vector $\mathbf{k}$ in the wave spectrum. The wider the angle the mean wind spans, the larger the fraction of wavenumber vectors that will be filtered by these critical levels, making resonant modes and trapped lee waves less likely to occur, for reasons that were explained in the preceding section. Among these three cases, Lin(II) has the smallest wind rotation angle, while this angle is largest for Lin(IV). As shown by the surface pressure fields displayed in Figure 8, the trapped lee wave pattern in cases $\mathrm{Lin}(\mathrm{II})$ and $\mathrm{Lin}(\mathrm{III})$ is stronger, as would be expected from the above reasoning, while for Lin(IV) it is substantially weaker and becomes harder to detect far downstream of the mountain. This article is protected by copyright. All rights reserved. (a)

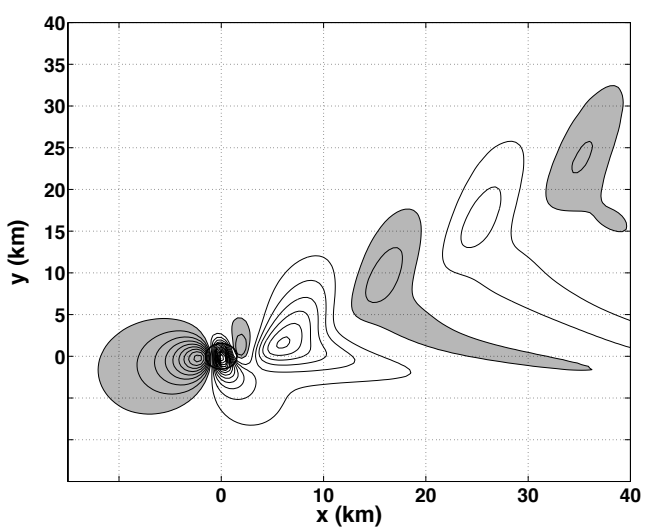

(b)

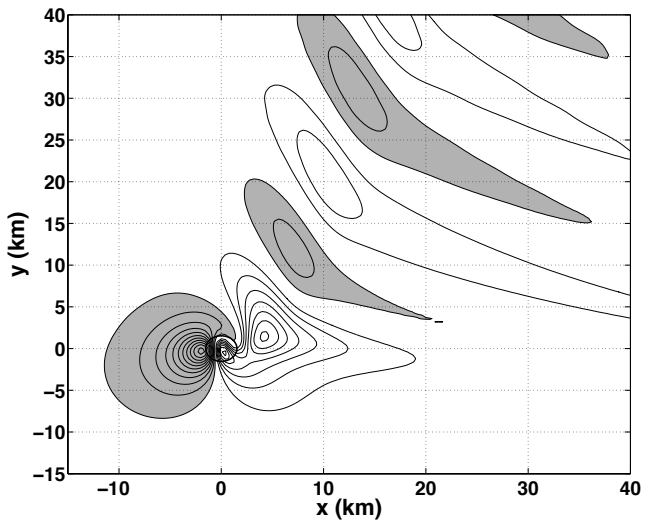

(c)

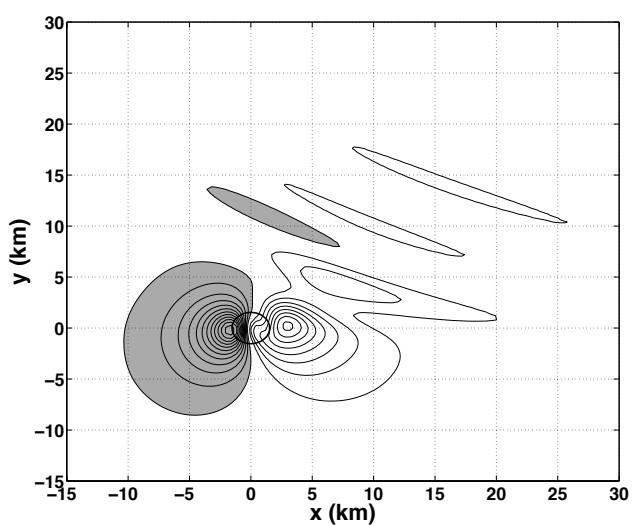

Figure 8. Fields of the normalized surface pressure $p(z=0) /\left(\rho_{0} U_{0} N h_{0}\right)$ for (a) $\operatorname{Lin}(\mathrm{II})$ with $\hat{a}=2$ and $R i=2$; (b) $\operatorname{Lin}(\mathrm{III})$ with $\hat{a}=1.5$ and $R i=2$; (c) $\operatorname{Lin}(\mathrm{IV})$ with $\hat{a}=2$ and $R i=2$. The contour spacing is 0.03 , and the zero contour is omitted for clarity. Shaded contours: positive values. The circle centred at $(0,0)$ denotes $0.5 h_{0}$ surface elevation.

The drag variation in these three cases is essentially a mixture of that for cases Lin4(I) and Lin(V) (Figure 9). In the hydrostatic limit, the oscillating drag behaviour with $R i$ is more pronounced for Lin(II), less so for Lin(III), and becomes weak for Lin(IV).

This agrees with the exact hydrostatic calculations of Teixeira et al. (2008a), and is consistent with the arguments used to interpret cases $\operatorname{Lin} 4(\mathrm{I})$ and $\operatorname{Lin}(\mathrm{V})$ above. In the non-hydrostatic limit, when $R i$ is large, the magnitude of $D_{x} / D_{0}$ for $\hat{a}=2$ or 1.5 gradually increases from case Lin(II) to Lin(IV), because Lin(II) exhibits more characteristics of Lin4(I) regarding critical level 
(a)

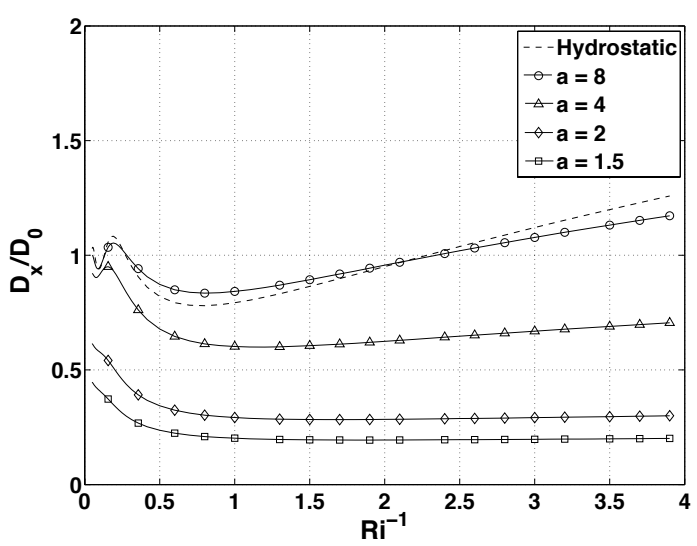

(c)

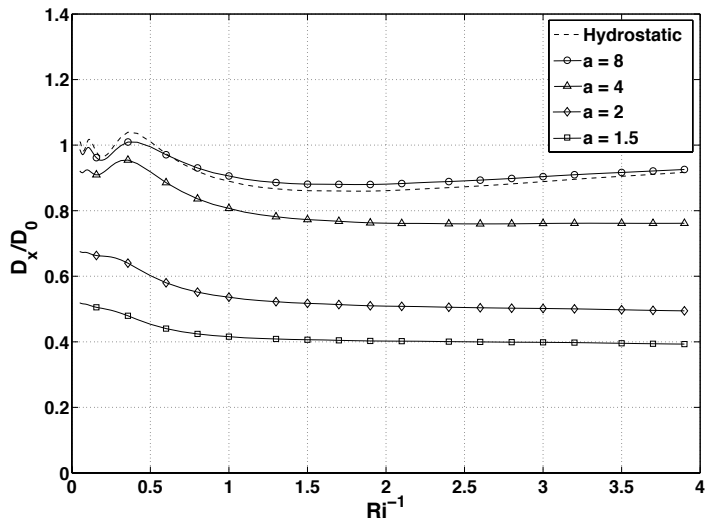

(e)

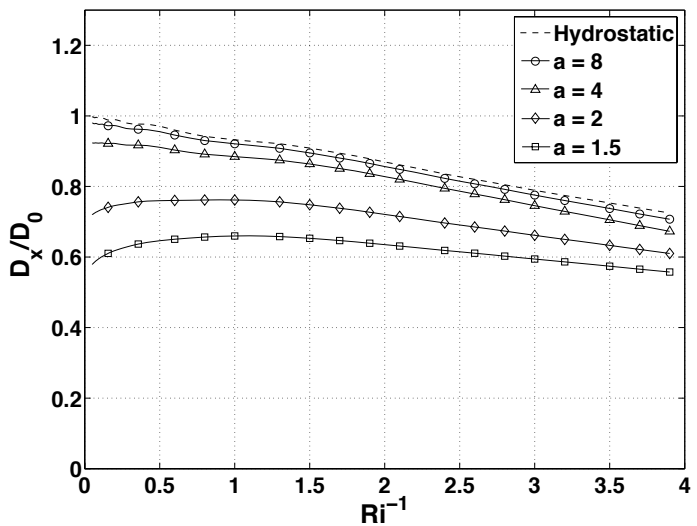

(b)

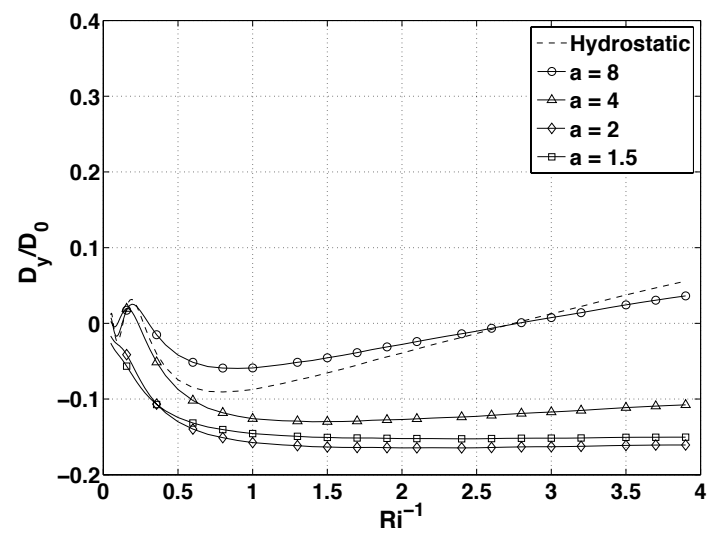

(d)

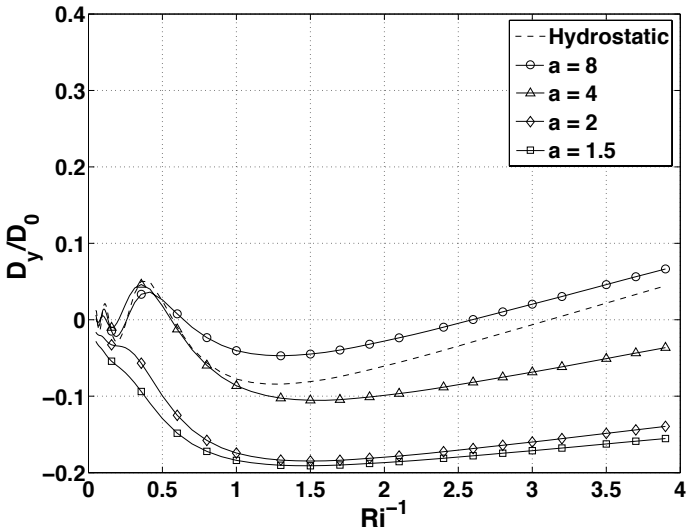

(f)

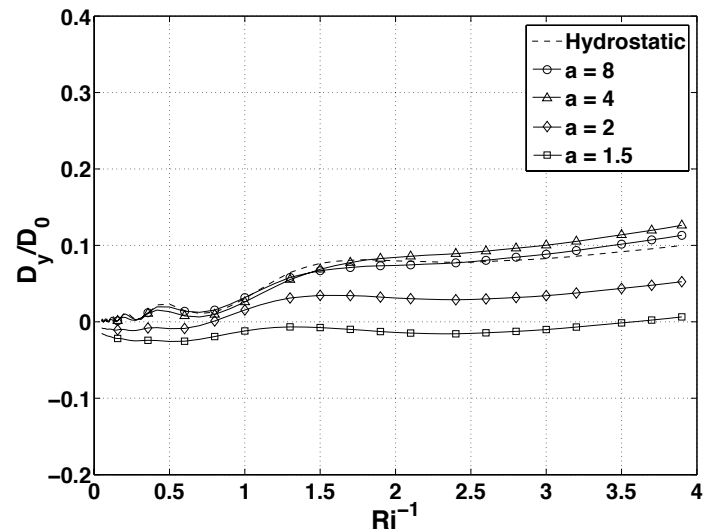

Figure 9. Normalized drag as a function of $R i^{-1}$. (a),(c),(e): $D_{x} / D_{0}$; (b),(d),(f): $D_{y} / D_{0}$; (a),(b): $\operatorname{Lin}(\mathrm{II}) ;$ (c),(d): $\operatorname{Lin}(\mathrm{III})$; (e),(f): $\operatorname{Lin}(\mathrm{IV})$. Curves for different values of $\hat{a}$ are labelled with different symbols (see legend). The symbol positions do not correspond to the data points.

filtering, while $\operatorname{Lin}(\mathrm{IV})$ is closer to $\operatorname{Lin}(\mathrm{V})$. This transition is more obvious by looking at the drag variation when both $\hat{a}$ and $R i$ are small. As Ri decreases, the magnitude of $D_{x} / D_{0}$ for Lin(II) decreases more rapidly than for Lin(III); while in Lin(IV), that magnitude increases before decreasing, as in Lin(V).

An important difference from the cases with unidirectional shear is the fact that the $y$-component of the drag, $D_{y} / D_{0}$, is now non-zero. Since all wind profiles considered here rotate anti-

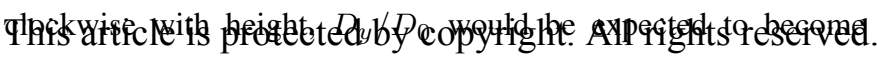

positive as $R i$ decreases. However, except for $\operatorname{Lin}(\mathrm{IV}), D_{y} / D_{0}$ becomes instead negative, except in very limited ranges of $R i$, and more so when non-hydrostatic effects become stronger, as shown in Figure 9(b),(d),(f). This misalignment of the drag with the surface wind in the direction opposite to the shear was also observed in the hydrostatic calculations of Teixeira et al. (2008a), being attributed to interference between upwardpropagating and downward-propagating waves reflected at $z=$ $H$. In non-hydrostatic conditions, it would be interesting to 
(a)

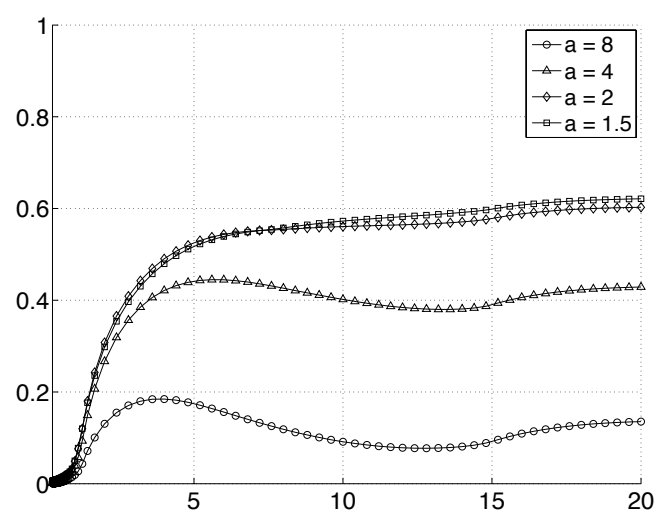

(c)

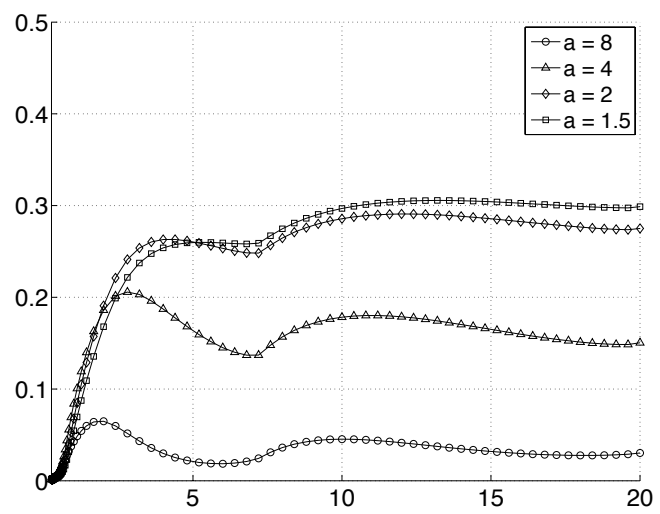

(b)

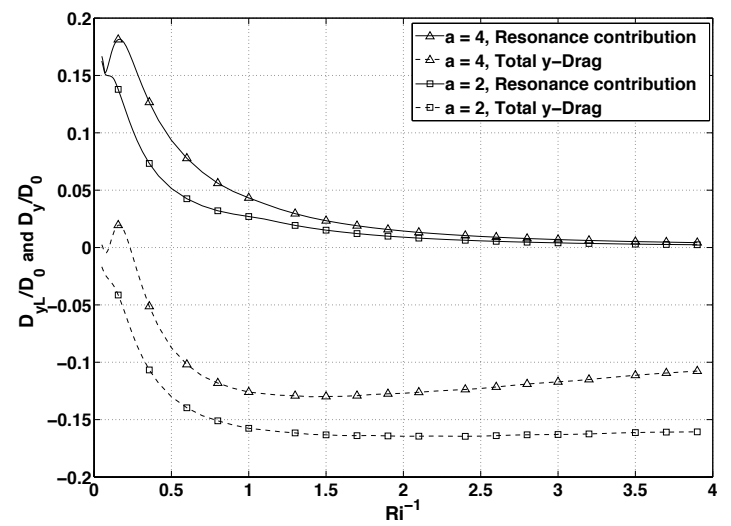

(d)

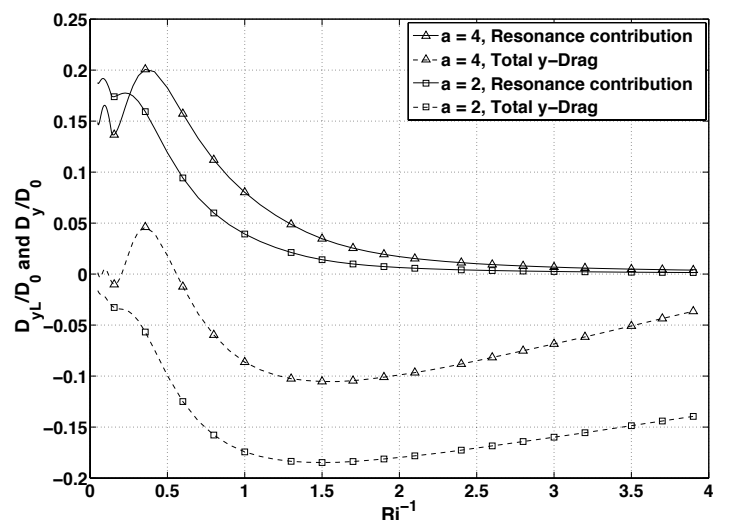

Figure 10. Normalized trapped lee wave drag. (a),(c): $D_{x L} / D_{x} ;$ (b),(d): $D_{y L} / D_{0}$ and $D_{y} / D_{0} ;$ (a),(b): Lin(II); (c),(d): Lin(III). Curves for different values of $\hat{a}$ are labelled with different symbols (see legend). The symbol positions do not correspond to the data points.

know whether trapped lee waves give any contribution to this phenomenon.

Figure 10 shows the fraction of the drag in the $x$ - direction that is due to trapped lee waves for Lin(II) and Lin(III), and the normalized $y$-component of the total drag and of the trapped lee wave drag, both as function of Ri. In Figure 10(a),(c) it can be seen that the fraction of $D_{x}$ attributable to trapped lee waves can be substantial, particularly for Lin(II), especially at high values of $R i$, and low values of $\hat{a}$. This is consistent with what was previously found for cases Lin4(I) and Lin8(I). Figure 10(b),(d) shows that the $y$ component of the trapped lee wave drag is always positive (consistent with the orientation of the trapped lee wave patterns in Figure 8(a),(b)), so the negative drag contribution must come totally from the waves partially reflected at $z=H$ (as in the hydrostatic case).

Figure 11 shows the angle made with the $x$-direction by the total surface drag for cases Lin(II) and Lin(III), for $\hat{a}=2$ and 1.5. The misalignment of the drag with the surface wind is significant in both Lin(II) and Lin(III) (corresponding to an angle as large

This article is protected by copyright. All rights reserved.

as $-38^{\circ}$ for $\hat{a}=1.5$ in Figure 11(a)). For such misalignment to occur, the angle spanned by the wind turning between $z=0$ and $z=H$ must not be too large, so that the filtering effect of critical levels is weak enough to allow reflected waves to have a substantial impact at the surface (cf. Figure 11(b)). The negative drag component in the $y$ direction can be understood by going back to Figure $8(\mathrm{~b})$, where it can be seen that the high pressure region on the left-hand (upstream) side of the mountain is connected with the nearest high pressure region on the lee side and wraps up in a clockwise-rotated pattern with the low pressure on the right-hand (downstream) side. This feature is not as clear in Figure $8(a)$, and cannot be seen in Figure $8(\mathrm{c})$, where the pressure perturbation is more symmetric about the $x$ axis, and thus the $y$ component of the drag is much weaker (in agreement with Figure $9(f))$.

\section{Concluding remarks}

A semi-analytical two-layer model of non-hydrostatic vertically sheared flow over a 3D axisymmetric mountain has been

(n)


(a)

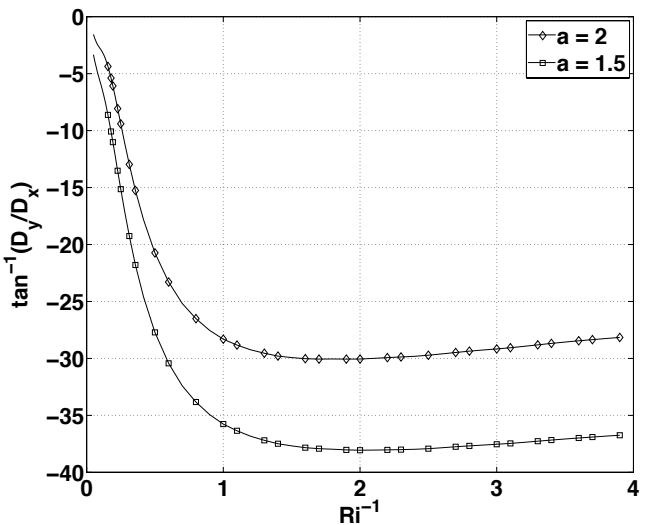

(b)

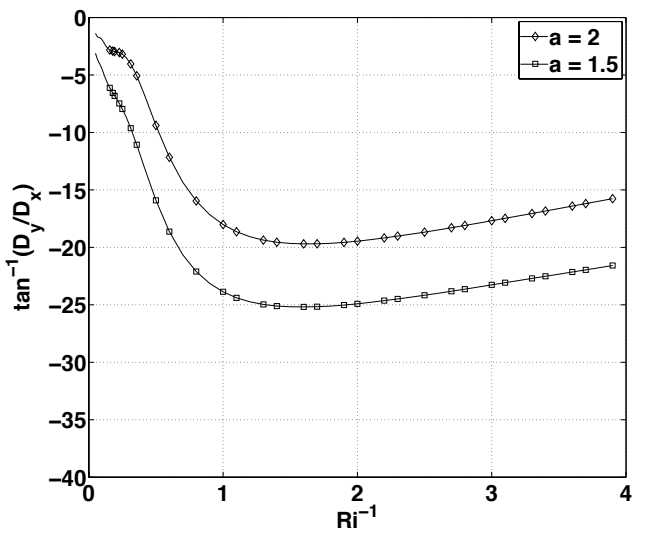

Figure 11. Misalignment angle of the total drag as a function of $R i^{-1}$ : (a) $\tan ^{-1}\left(D_{y} / D_{x}\right)$ for Lin(II), (b) $\tan ^{-1}\left(D_{y} / D_{x}\right)$ for Lin(III). Curves for different values of $\hat{a}$ are labelled with different symbols (see legend). The symbol positions do not correspond to the data points.

developed, to assess the impact of non-hydrostatic effects and resonant trapped lee wave modes on the surface drag exerted by 1 mountain waves. The model, which is based on linear theory and developed in spectral space, handles both the continuous spectrum of the waves that propagate upward to infinity in the upper layer and the discrete spectrum of waves trapped in the lower constantshear layer. The surface pressure field and drag were calculated in conditions ranging from hydrostatic (addressed previously by Teixeira et al. (2008a)) to strongly non-hydrostatic.

The model was first verified by comparing its predicted vertical velocity perturbation in a situation dominated by 3D trapped lee waves against numerical simulations of the same situation produced by previous authors (Broutman et al.,2003). The surface drag contribution from resonant wave modes was then analyzed in a 2D framework. It was found that trapped lee waves always provide a positive contribution to the surface drag by deepening the low pressure anomaly on the lee side of the mountain (in agreement with the findings of Teixeira et al. (2013a) and Teixeira et al. (2013b) for simpler atmospheres). For a model with a single, constant-shear layer extending indefinitely, the near-field pressure was shown to be symmetric about the mountain and to have no impact on the drag, which then only receives contributions from the resonant trapped lee wave modes.

Wind profiles with five different shear directions were studied, among which Lin4(I), Lin8(I) and Lin(V) are unidirectional, while Lin(II), Lin(III) and Lin(IV) have directional shear. The drag variation with the Richardson number in the hydrostatic limit agrees well with the results of previous exact calculations by Teixeira et al. (2008a), lending credence to the numerical approach employed here. As the flow becomes more nonhydrostatic, the generation of the shortest waves by the mountain is inhibited, and the shear in the wind profile also causes total wave reflection in the lower atmospheric layer in addition to the partial wave reflections that take place at the interface between the two layers. Both reasons can lead, through destructive wave interference, to a strong reduction in the surface drag (as large as $30-50 \%$ for high $R i$ and about $50-75 \%$ for low $R i$ ). The drag associated with trapped lee wave modes seems unable to compensate for this decrease, unlike in Teixeira et al. (2013a) and Teixeira et al. (2013b).

For the flows with unidirectional shear, the drag reduction is considerably stronger for forward shear than for the backward shear, since the presence of a total critical level in the latter case prevents the reflected waves from reaching the surface. The drag reduction is then mainly caused by the direct effect of shear on the wave structure (Grubišić and Smolarkiewicz,1997), or by the existence of waves that are already evanescent at the surface. In flows with forward shear, the drag reduction is also enhanced by destructive interference with downward propagating, totally or partially reflected waves.

As the Richardson number drops below 1, trapped lee wave modes become weak or absent, so contributions to the drag come essentially from the near-field pressure. By increasing the thickness of the lower layer (i.e. increasing $\left|\mathbf{U}_{H}\right| /\left|\mathbf{U}_{0}\right|$ ), leakage of gravity waves into the upper layer is reduced, leading to an increasingly symmetric surface pressure distribution, and hence a low drag value. For the directionally sheared flows considered here, the larger the angle spanned by the wind vector, the larger the fraction of the gravity waves that are filtered by critical levels,

This article is protected by copyright. All rights reserved. 
reducing the strength of partially reflected and trapped lee waves.

Hence as one shifts from Lin(II) to Lin(III) and Lin(IV), the drag behaviour begins by resembling that of $\operatorname{Lin} 4$ (I) to finally resemble more closely Lin(V).

A striking result obtained in directionally sheared flows is that the drag may have a misalignment with the surface wind in the direction opposite to that of the shear by an angle as large as $38^{\circ}$. This effect was noted by Teixeira et al. (2008a) for hydrostatic conditions, but it becomes stronger as the flow becomes more nonhydrostatic, being presumaby attributable to interference caused by reflected waves. The effect appears to be totally due to nontrapped waves, since trapped lee waves always counteract it by contributing to the drag roughly along the direction of the shear vector.

While the present results do not suggest a substantial total drag enhancement due to non-hydroastatic effects, at least in the linear wave regime, they corroborate the idea that a large fraction of the drag may be produced by trapped lee waves. This corresponds to a reaction force that is exerted on the lower atmosphere, sometimes with a direction quite different to that of the drag associated with vertically propagating waves (as was seen above). A representation of this currently-neglected effect in orographic drag parametrizations seems therefore necessary.

\section{Acknowledgements}

M.A.C.T. acknowledges the financial support of the Portuguese Science Foundation (FCT) under Grant PTDC/CTEATM/122501/2010 and of the European Commission, under Marie Curie Career Integration Grant GLIMFLO, contract PCIG13-GA-2013-618016.

\section{Appendix A. Solutions corresponding to upward and downward propagating waves using Bessel functions}

In order to justify the form taken by the wave solutions (8)-(11) and (12)-(13), we present here a generalization to 3D flow of the approach developed by Booker and Bretherton (1967) (see also Grubišić and Smolarkiewicz,1997). It is useful to depart from the hydrostatic limit and then extend the results to nonhydrostatic conditions. A hydrostatic non-stationary version of the Talyor-Goldstein equation for a constant-shear flow can be written This article is protected by copyright. All rights reserved.
(Booker and Bretherton,1967)

$$
\hat{w}^{\prime \prime}+\left[\frac{N^{2} k_{12}^{2}}{\{(\mathbf{c}-\mathbf{U}) \cdot \mathbf{k}\}^{2}}\right] \hat{w}=0,
$$

where the non-zero phase velocity $\mathbf{c}$ takes the form $\mathbf{c}=\mathbf{c}_{r}+i \mathbf{c}_{i}$, with its imaginary part $\mathbf{c}_{i}$ assumed to be small. In the vicinity of the critical level, the background wind $\mathbf{U}$ may be expanded as

$$
\mathbf{U}(z)=\mathbf{c}_{r}+\mathbf{U}^{\prime}\left(z_{c}\right)\left(z-z_{c}\right)+\frac{1}{2} \mathbf{U}^{\prime \prime}\left(z_{c}\right)\left(z-z_{c}\right)^{2}+\ldots
$$

Substituting (A2) into (A1), we have

$$
\hat{w}^{\prime \prime}+\left(\frac{N^{2} k_{12}^{2}}{\left[\left\{i \mathbf{c}_{i}-\mathbf{U}_{c}^{\prime}\left(z-z_{c}\right)\right\} \cdot \mathbf{k}\right]^{2}}\right) \hat{w}=0,
$$

(where $\mathbf{U}_{c}^{\prime}=\mathbf{U}^{\prime}\left(z_{c}\right)$ ), because all higher derivatives of $\mathbf{U}$ are zero. By dividing the numerator and denominator of the fraction by $\left(\mathbf{U}_{c}^{\prime} \cdot \mathbf{k}\right)^{2}$, we obtain

$$
\hat{w}^{\prime \prime}+\left[\frac{\tilde{R} i}{\left\{\left(z-z_{c}\right)-\frac{i \mathbf{c}_{i} \cdot \mathbf{k}}{\mathbf{U}_{c}^{\prime} \cdot \mathbf{k}}\right\}^{2}}\right] \hat{w}=0
$$

Here, we assume that $\mathbf{c}_{i} \cdot \mathbf{k}>0$, that is, the dissipation is always along the chosen horizontal wavenumber vector $\mathbf{k}$. By using a Frobenius expansion, the solution to (A4) is found to be

$$
\begin{array}{r}
\hat{w}\left(z-z_{c}\right)=D_{1}\left(z-z_{c}-i \frac{\mathbf{c}_{i} \cdot \mathbf{k}}{\mathbf{U}_{c}^{\prime} \cdot \mathbf{k}}\right)^{\lambda_{+}}+ \\
D_{2}\left(z-z_{c}-i \frac{\mathbf{c}_{i} \cdot \mathbf{k}}{\mathbf{U}_{c}^{\prime} \cdot \mathbf{k}}\right)^{\lambda_{-}}
\end{array}
$$

where $D_{1}$ and $D_{2}$ are complex coefficients, and $\lambda_{ \pm}=1 / 2 \pm i \mu$.

If $\mathbf{U}_{c}^{\prime} \cdot \mathbf{k}>0$, then as $\left|\mathbf{c}_{i}\right| \rightarrow 0$, for $z<z_{c}$ we have

$$
z-z_{c}=-\left|z-z_{c}\right|=e^{-i \pi}\left|z-z_{c}\right|
$$

Therefore,

$$
\begin{aligned}
\left(z-z_{c}\right)^{\lambda_{ \pm}} & =e^{-i \pi \lambda_{ \pm}}\left|z-z_{c}\right|^{\lambda_{ \pm}} \\
& =-i \sqrt{\left|z-z_{c}\right|} e^{ \pm \pi \mu}\left(\left|z-z_{c}\right|\right)^{ \pm i \mu}
\end{aligned}
$$


Similarly, if $\mathbf{U}_{c}^{\prime} \cdot \mathbf{k}<0$, we have instead

$$
\begin{aligned}
\left(z-z_{c}\right)^{\lambda_{ \pm}} & =e^{i \pi \lambda_{ \pm}}\left|z-z_{c}\right|^{\lambda_{ \pm}} \\
& =i \sqrt{\left|z-z_{c}\right|} e^{\mp \pi \mu}\left(\left|z-z_{c}\right|\right)^{ \pm i \mu} .
\end{aligned}
$$

Booker and Bretherton (1967) showed, by investigating the vertical energy fluxes, that solutions with the factor $e^{\pi \mu}$ below the critical level are associated with upward energy propagation, while those with the factor $e^{-\pi \mu}$ are associated with downward energy propagation (as seems intuitive). Therefore, in the vicinity of the critical level, the upward-propagating wave solution can be written

$$
\begin{aligned}
& \hat{w}^{\uparrow}\left(z>z_{c}\right) \propto\left|z-z_{c}\right|^{\lambda_{(\mathrm{sgn})}} \\
& \hat{w}^{\uparrow}\left(z<z_{c}\right) \propto-(\operatorname{sgn}) i e^{\pi \mu}\left|z-z_{c}\right|^{\lambda_{(\mathrm{sgn})}},
\end{aligned}
$$

where $\operatorname{sgn}=\operatorname{sign}\left(\mathbf{U}_{c}^{\prime} \cdot \mathbf{k}\right)$. Similarly, the downward-propagating wave solution takes the form

$$
\begin{aligned}
& \hat{w}^{\downarrow}\left(z>z_{c}\right) \propto\left|z-z_{c}\right|^{\lambda_{(-\mathrm{sgn})}} \\
& \hat{w}^{\downarrow}\left(z<z_{c}\right) \propto-(\operatorname{sgn}) i e^{-\pi \mu}\left|z-z_{c}\right|^{\lambda_{(-\mathrm{sgn})}} .
\end{aligned}
$$

For non-hydrostatic conditions, the solution for $z>z_{c}$ can be expressed in terms of Bessel $I$ functions of imaginary order $i \mu$ as $\sqrt{z-z_{c}} I_{ \pm i \mu}\left(k_{12}\left(z-z_{c}\right)\right)$. Near the origin (i.e. for $z \approx z_{c}$ ), this solution exhibits the following asymptotic behaviour

$$
\sqrt{z-z_{c}} I_{ \pm i \mu}\left(k_{12}\left(z-z_{c}\right)\right) \propto\left(z-z_{c}\right)^{\lambda_{ \pm}}
$$

which is consistent with the fact that the flow is hydrostatic near critical levels. The Bessel $I$ function also has the property that

$$
I_{i \mu}\left(k_{12} \xi\right)=I_{i \mu}\left(k_{12}|\xi|\right) \exp ( \pm \pi \mu)
$$

for negative $\xi$ and positive $k_{12}$ (Abramowitz and Stegun (1972), Eq. (9.6.30)). This, together with the fact that the non-hydrostatic solution asymptotically approaches the hydrostatic solution near the critical level, allows us to express the upward-propagating

This article is protected by copyright. All rights reserved. wave solution in the following form

$$
\begin{aligned}
& \hat{w}^{\uparrow}\left(z>z_{c}\right) \propto \sqrt{\xi} I_{i(\operatorname{sgn}) \mu}\left(k_{12} \xi\right) \\
& \hat{w}^{\uparrow}\left(z<z_{c}\right) \propto-i(\operatorname{sgn}) e^{\pi \mu} \sqrt{|\xi|} I_{i(\operatorname{sgn}) \mu}\left(k_{12}|\xi|\right),
\end{aligned}
$$

while the downward-propagating solution is written as

$$
\begin{aligned}
& \hat{w}^{\downarrow}\left(z>z_{c}\right) \propto \sqrt{\xi} I_{-i(\operatorname{sgn}) \mu}\left(k_{12} \xi\right) \\
& \hat{w}^{\downarrow}\left(z<z_{c}\right) \propto-i(\operatorname{sgn}) e^{-\pi \mu} \sqrt{|\xi|} I_{-i(\operatorname{sgn}) \mu}\left(k_{12}|\xi|\right) .
\end{aligned}
$$

Other solution forms using the Bessel $K$ function and the associated function $L$ have been employed by Wurtele et al. (1987), because they are more appropriate for computational purposes, as they are purely exponentially decaying or growing. (Note that, unlike in a constant-wind atmosphere, for the present linear wind profile, upward/downward-propagating solutions do not reduce to exponentially decaying/growing solutions, respectively, when the waves become evanescent). By using the relations

$$
\begin{aligned}
L_{i \mu}(x) & =\frac{\pi}{2} \frac{I_{i \mu}(x)+I_{-i \mu}(x)}{\sinh (\mu \pi)} \\
K_{i \mu}(x) & =\frac{i \pi}{2} \frac{I_{i \mu}(x)-I_{-i \mu}(x)}{\sinh (\mu \pi)},
\end{aligned}
$$

where $x$ is real, a definition of the Bessel $I$ function in terms of the $K$ and $L$ functions may be obtained, as follows:

$$
I_{ \pm i(\operatorname{sgn}) \mu}\left(k_{12} \xi\right) \propto\left(L_{i \mu}\left(k_{12} \xi\right) \mp i(\operatorname{sgn}) K_{i \mu}\left(k_{12} \xi\right)\right)
$$

Using this relation, the final form for the solutions expressed by (12) and (13) may be reached.

\section{Appendix B. Symmetry of the near-field pressure} perturbation with respect to the mountain

We aim to show here that when it is assumed that the wind profile has a forward shear extending indefinitely, the near-field pressure perturbation is symmetric with respect to the mountain, and hence does not contribute to the surface drag.

Note that the contours of integration $C_{1}$ and $C_{2}$ displayed in Figure 2 can be arbitrarily chosen, as long as they lie on both sides of the complex plane. For convenience, $C_{2}$ will be chosen to be 
the complex conjugate of $C_{1}$, i.e. $C_{2}=\overline{C_{1}}$. If the solution $\hat{\phi}$ (see (34)) satisfies the following two properties

$$
\hat{\phi}\left(\overline{k_{1}}, z\right)=\overline{\hat{\phi}\left(k_{1}, z\right)} \quad \text { and } \quad \hat{\phi}^{\prime}\left(\overline{k_{1}}, z\right)=\overline{\hat{\phi}^{\prime}\left(k_{1}, z\right)},
$$

then for $x<0$ (i.e. $x=-|x|$ ), we have

$$
\begin{aligned}
& \operatorname{Re}\left(\int_{C_{2}} \frac{\hat{\phi}^{\prime}\left(k_{1}, z=0\right)}{\hat{\phi}\left(k_{1}, z=0\right)} e^{i k_{1} x-a k_{1}} d k_{1}\right) \\
= & \operatorname{Re}\left(\int_{C_{1}} \frac{\hat{\phi}^{\prime}\left(\overline{k_{1}}, z=0\right)}{\hat{\phi}\left(\overline{k_{1}}, z=0\right)} e^{i \overline{k_{1}} x-a \overline{k_{1}}} d \overline{k_{1}}\right) \\
= & \operatorname{Re}\left(\int_{C_{1}} \overline{\left(\frac{\hat{\phi}^{\prime}\left(k_{1}, z=0\right)}{\hat{\phi}\left(k_{1}, z=0\right)}\right)} e^{-i \overline{k_{1}}|x|-a \overline{k_{1}}} d \overline{k_{1}}\right) \\
= & \operatorname{Re}\left(\int_{C_{1}} \frac{\overline{\phi^{\prime}\left(k_{1}, z=0\right)}}{\hat{\phi}\left(k_{1}, z=0\right)} e^{i k_{1}|x|-a k_{1}} d k_{1}\right) \\
= & \operatorname{Re}\left(\int_{C_{1}} \frac{\hat{\phi}^{\prime}\left(k_{1}, z=0\right)}{\hat{\phi}\left(k_{1}, z=0\right)} e^{i k_{1}|x|-a k_{1}} d k_{1}\right) .
\end{aligned}
$$

This shows that performing the integration along the path $C_{2}$ for some $x<0$ is equivalent to performing it along $C_{1}$ for $|x|$. Hence, if leakage of gravity waves to the upper atmosphere is impossible, then the pressure caused by non-resonant wave modes is symmetric with respect to the mountain, which means the corresponding contribution to the surface drag is zero. The last step in (B3) uses the fact that

$$
\operatorname{Re}\left(\int_{C} \overline{f(z)}\right)=\operatorname{Re}\left(\int_{C} f(z)\right)
$$

for any complex contour $C$ and smooth complex function $f(z)$.

For forward shear extending indefinitely, the physical solution satisfying the boundedness condition uses the Bessel $K$ function, $\sqrt{z-z_{c}} K_{i \mu}\left(k_{1}\left(z-z_{c}\right)\right)$. It turns out that this expression indeed satisfies the two conditions expressed by (B1). However, if leakage of gravity waves to the upper atmosphere is possible (as happens in a two-layer model), then $\hat{\phi}$ is not simply $\sqrt{z-z_{c}} K_{i \mu}\left(k_{1}\left(z-z_{c}\right)\right)$, but contains an additional imaginary part, i.e. $\hat{\phi}=\sqrt{z-z_{c}}\left(\tilde{A} K_{i \mu}\left(k_{1}\left(z-z_{c}\right)\right)+\tilde{B} L_{i \mu}\left(k_{1}\left(z-z_{c}\right)\right)\right)$, where $\tilde{A}$ and $\tilde{B}$ are non-zero complex constants. Then, $\hat{\phi}$ does not satisfy (B1) and the near-field pressure will not be symmetric.

\section{References}

Abramowitz M, Stegun IA. 1972. Handbook of mathamatical functions: with formulas, graphs, and mathematical tables. Dover Publications. This article is protected by copyright. All rights reserved.
Booker JR, Bretherton FP. 1967. The critical layer for internal gravity waves in a shear flow. J. Fluid Mech. 27: 513-539.

Bretherton FP. 1969. Momentum transport by gravity waves. Quart. J. Roy. Meteor. Soc. 95: 213-243.

Broad AS. 1995. Linear theory of momentum fluxes in 3-d flows with turning of the mean wind with height. Quart. J. Roy. Meteor. Soc. 121: 1891-1902.

Broutman D, Rottman JW, Eckermann SD. 2003. A simplified fourier method for nonhydrostatic mountain waves. J. Atmos. Sci. 60: 2686-2696.

Durran DR. 1986. Another look at downslope windstorms. part i: The development of analogs to supercritical flow in an infinitely deep, continuously stratified fluid J. Atmos. Sci. 43: 2527-2543.

Gill AE. 1982. Atmosphere-ocean dynamics. Academic Press.

Grubišić V, Smolarkiewicz PK. 1997. The effect of critical levels on 3d orographic flows: linear regime. J. Atmos. Sci. 54: 1943-1960.

Grubišić V, Stiperski I. 2009. Lee-wave resonances over double bell-shaped obstacles. J. Atmos. Sci. 66: 1205-1228.

Jiang Q, Smith RB, Doyle JD. 2008. Impact of the atmospheric boundary layer on mountain waves. J. Atmos. Sci. 65: 592-608.

Keller TL. 1994. Implications of the hydrostatic assumption on atmospheric gravity waves. J. Atmos. Sci. 51: 1915-1929.

Kim YJ, Doyle JD. 2005. Extension of an orographic-drag parametrization scheme to incorporate orographic anisotropy and flow blocking. Quart. J. Roy. Meteor. Soc. 131: 1893-1921.

Lin YL. 2007. Mesoscale dynamics. Cambridge University Press.

Lott F. 2007. The reflection of a stationary gravity wave by a viscous boundary layer. J. Atmos. Sci. 64: 3363-3371.

Lott F, Miller MJ. 1997. A new subgrid-scale orographic drag parametrization: its formulation and testing. Quart. J. Roy. Meteor. Soc. 123: 101-127.

Miranda PMA, James IN. 1992. Non-linear three dimensional effects on the wave drag: splitting flow and breaking waves. Quart. J. Roy. Meteor. Soc. 118: 10571081.

Miranda PMA, Valente MA. 1997. Critical level resonance in three-dimensional flow past isolated mountains. J. Atmos. Sci. 54: 1574-1588.

Mitchell RM, Cechet RP, Turner PJ, Elsum CC. 1990. Observation and interpretation of wave clouds over macquaire island. Quart. J. Roy. Meteor. Soc. 116: $741-752$

Nappo CJ. 2012. An introduction to atmospheric gravity waves - second edition Academic Press.

NOAA. 1976. Us standard atmosphere 1976. Technical report, National Oceanic and Atmospheric Administration, US Government Printing Office, Washington, D.C.

Ólafsson H, Bougeault P. 1996. Nonlinear flow past an elliptic mountain ridge. $J$. Atmos. Sci. 53: 2465-2489.

Ólafsson H, Bougeault P. 1997. The effect of rotation and surface friction on orographic drag. J. Atmos. Sci. 54: 193-210.

Sawyer JS. 1960. Numerical calculation of the displacements of a stratified airstream crossing a ridge of small height. Quart. J. Roy. Meteor. Soc. 86: 326-345.

Scorer RS. 1949. Theory of waves in the lee of mountains. Quart. J. Roy. Meteor Soc. 75: 41-56.

Shutts G. 1995. Gravity-wave drag parametrization over complex terrain: The effect of critical-level absorption in directional wind-shear. Quart. J. Roy. Meteor. Soc. 121: $1005-1021$. 
Shutts GJ, Gadian A. 1999. Numerical simulations of orographic gravity waves in flows which back with height. Quart. J. Roy. Meteor. Soc. 125: 2743-2765.

Smith RB. 1976. The generation of lee waves by the blue ridge. J. Atmos. Sci. 33: $507-519$.

Stensrud DJ. 2009. Parametrization schemes: Keys to understanding numerical weather prediction models. Cambridge University Press.

Stiperski I, Grubišić V. 2011. Trapped lee wave interference in presence of surface friction. J. Atmos. Sci. 68: 918-936.

Teixeira MAC, Argaín JL, Miranda PMA. 2013a. Drag produced by trapped lee waves and propagating mountain and propagating mountain waves in a two-layer atmosphere. Quart. J. Roy. Meteor. Soc. 139: 964-981.

Teixeira MAC, Argaín JL, Miranda PMA. 2013b. Orographic drag associated with lee waves trapped at an inversion. J. Atmos. Sci. 70: 2930-2947.

Teixeira MAC, Miranda PMA. 2004. The effect of wind shear and curvature on the gravity wave drag produced by a ridge. J. Atmos. Sci. 61: 2638-2643.
Teixeira MAC, Miranda PMA. 2006. A linear model of gravity wave drag for hydrostatic sheared flow over elliptical mountains. Quart. J. Roy. Meteor. Soc. 132: $2439-2458$.

Teixeira MAC, Miranda PMA, Argaín JL. 2008a. Mountain waves in two-layer sheared flows: critical level effects, wave reflection and drag enhancement. $J$. Atmos. Sci. 65: 1912-1926

Teixeira MAC, Miranda PMA, Cardoso RM. 2008b. Asymptotic gravity wave drag expressions for non-hydrostatic rotating flow over a ridge. Quart. J. Roy. Meteor. Soc. 134: 271-276.

Teixeira MAC, Miranda PMA, Valente MA. 2004. An analytical model of mountain wave drag for wind profiles with shear and curvature. J. Atmos. Sci. 61: 10401054.

Wurtele MG, Sharman RD, Keller TL. 1987. Analysis and simulations of a troposphere-stratosphere gravity wave model. part i. J. Atmos. Sci. 44: 32693281 